TRANSACTIONS OF THE

AMERICAN MATHEMATICAL SOCIETY

Volume 361, Number 10, October 2009, Pages 5505-5537

S 0002-9947(09)04720-5

Article electronically published on April 20, 2009

\title{
QUANTUM COHOMOLOGY RINGS OF LAGRANGIAN AND ORTHOGONAL GRASSMANNIANS AND TOTAL POSITIVITY
}

\author{
DAEWOONG CHEONG
}

\begin{abstract}
We verify in an elementary way a result of Peterson for the maximal orthogonal and Lagrangian Grassmannians, and then find Vafa-Intriligator type formulas which compute their 3-point, genus zero Gromov-Witten invariants. Finally we study the total positivity of the related Peterson's varieties and show that Rietsch's conjecture about the total positivity holds for these cases.
\end{abstract}

\section{INTRODUCTION}

The (small) quantum cohomology ring $q H^{*}(X)$ of a projective complex manifold $X$ is a certain deformation of the classical cohomology ring of $X$. The additive structure of the ring $q H^{*}(X)$ is the same as that of the classical one, but the multiplicative one is deformed from that of the classical cohomology; the structure constants for the quantum multiplication are the 3-point Gromov-Witten invariants of genus 0, which count equivalence classes of certain rational curves in $X$.

In 1997, D. Peterson announced in his MIT lectures that the (complexified) quantum cohomology ring $q H_{\mathbb{C}}^{*}(G / P)$ of a homogeneous manifold $G / P$ is isomorphic to the coordinate ring of a subvariety $\mathcal{Y}_{P}$ of the so-called Peterson variety $\mathcal{Y} \subset G^{\vee} / B^{\vee}$ (see $\oint 3.3$ ), where $G^{\vee}$ is the Langlands dual of $G$, and $B^{\vee}$ is a Borel subgroup of $G^{\vee}$. The proof of Peterson's general theorem remains unpublished. In the general case it is not easy to see the quantum cohomology rings concretely via those isomorphisms, because the varieties $\mathcal{Y}_{P}$ are defined implicitly. However if $P$ is a minuscule parabolic subgroup, then we can replace the variety $\mathcal{Y}_{P}$ by an isomorphic subvariety $\mathcal{V}_{P}$ of $G^{\vee}$ whose coordinate ring is more explicit (see 33.3 ).

In [19], Rietsch verified the result of Peterson for the Grassmannian manifolds $G L_{n}(\mathbb{C}) / P$ in type $A$ by constructing in an elementary way an isomorphism between the coordinate ring of $\mathcal{V}_{P}$ and the quantum cohomology ring of $G L_{n}(\mathbb{C}) / P$. In addition, using this isomorphism, Rietsch derived for Grassmannian manifolds in type $A$ the Vafa-Intriligator formula, which gives the Gromov-Witten invariants by evaluating certain symmetric functions on some roots of unity. Then Rietsch identified the totally nonnegative elements in the variety $\mathcal{V}_{P}$ and characterized these elements via the nonnegativity of Schubert basis elements on $\mathcal{V}_{P}$, which are determined by the Schur polynomials.

Received by the editors July 24, 2007 and, in revised form, December 20, 2007.

2000 Mathematics Subject Classification. Primary 14N35, 20 G05.

(C)2009 American Mathematical Society Reverts to public domain 28 years from publication 
In this paper, we find analogues of Rietsch's results in the other classical Lie types. Consider a complex vector space $V$ of dimension $N$ with a nondegenerate (symmetric or skew-symmetric) bilinear form $Q$. For $Q$ a symmetric bilinear form and $N=2 n+2$, let $O G^{e}(n)$ be one component of the parameter space of maximal isotropic subspaces of $V$, and for $Q$ a symmetric bilinear form and $N=2 n+1$, let $O G^{o}(n)$ be the parameter space of maximal isotropic subspaces of $V$. Recall that subspace $W$ of $V$ is called isotropic if $Q(v, w)=0$ for all $v, w \in W$. For $N=2 n$ and $Q$ a skew-symmetric bilinear form, we denote by $L G(n)$ the parameter space of the Lagrangian (i.e., maximal isotropic) subspaces of $V$. If we denote by $P_{n}$ all the parabolic subgroups corresponding to 'right end roots' in the Dynkin diagrams for the classical algebraic groups with rank $n$, then $O G^{o}(n)=S O_{2 n+1}(\mathbb{C}) / P_{n}$, $L G(n)=S p_{2 n}(\mathbb{C}) / P_{n}$ and $O G^{e}(n)=S O_{2 n+2}(\mathbb{C}) / P_{n+1}$. It is well known that $O G^{e}(n)$ is isomorphic to $O G^{o}(n)$.

The cohomology ring $H^{*}(L G(n))$ is generated by the Schubert classes $\sigma_{\lambda}$, with $\lambda$ strict partitions $\lambda=\left(\lambda_{1}, \ldots, \lambda_{n}\right)$ (see $\$ 2.5$ ) such that $\lambda_{1} \leq n$, and the quantum cohomology ring $q H^{*}(L G(n))$ is isomorphic, as a $\mathbb{Z}[q]$-module, to $H^{*}(L G(n)) \otimes \mathbb{Z}[q]$. The multiplication of $q H^{*}(L G(n))$ is given by

$$
\sigma_{\lambda} \cdot \sigma_{\mu}=\sum_{\nu, d}\left\langle\sigma_{\lambda}, \sigma_{\mu}, \sigma_{\hat{\nu}}\right\rangle_{d} \sigma_{\nu} q^{d}
$$

where the sum is taken over all strict partitions $\nu$ with $\nu_{1} \leq n$ and nonnegative integers $d, \hat{\nu}$ denotes the partition whose parts complement the parts of $\nu$ in the set $\{1, \ldots, n\}$, so that the classes $\sigma_{\nu}$ and $\sigma_{\hat{\nu}}$ are Poincaré dual to each other, and $\left\langle\sigma_{\lambda}, \sigma_{\mu}, \sigma_{\hat{\nu}}\right\rangle_{d}$ are Gromov-Witten invariants (see 33.1 ).

Similarly, the cohomology ring $H^{*}\left(O G^{e}(n)\right)$ is generated by the Schubert classes $\tau_{\lambda}$ with $\lambda=\left(\lambda_{1}, \ldots, \lambda_{n}\right)$ strict partitions such that $\lambda_{1} \leq n$. The quantum cohomology ring $q H^{*}\left(O G^{e}(n)\right)$ is isomorphic, as a $\mathbb{Z}[q]$-module, to $H^{*}\left(O G^{e}(n)\right) \otimes \mathbb{Z}[q]$. The multiplication of $q H^{*}\left(O G^{e}(n)\right)$ is given by

$$
\tau_{\lambda} \cdot \tau_{\mu}=\sum_{\nu, k}\left\langle\tau_{\lambda}, \tau_{\mu}, \tau_{\hat{\nu}}\right\rangle_{k} \tau_{\nu} q^{k}
$$

where the sum is taken over all strict partitions $\nu$ with $\nu_{1} \leq n$ and nonnegative integers $k$, and $\left\langle\tau_{\lambda}, \tau_{\mu}, \tau_{\hat{\nu}}\right\rangle_{k}$ are Gromov-Witten invariants (see $\$ 3.2$ ).

To a triple $\left(G, G^{\vee}, P\right)$ with $P$ a parabolic subgroup of $G$, we associate a closed subvariety $\mathcal{V}_{P}$ of $G^{\vee}$ (see 3.3 . We write $\mathcal{V}_{n}^{C}, \mathcal{V}_{n}^{B}$ and $\mathcal{V}_{n+1}^{D}$ rather than $\mathcal{V}_{P}$ for the triples $\left(S O_{2 n+1}(\mathbb{C}), S p_{2 n}(\mathbb{C}), P_{n}\right), \quad\left(S p_{2 n}(\mathbb{C}), S O_{2 n+1}(\mathbb{C}), P_{n}\right)$ and $\left(S O_{2 n+2}(\mathbb{C}), S O_{2 n+2}(\mathbb{C}), P_{n+1}\right)$, respectively. Let $\mathcal{O}\left(\mathcal{V}_{P}\right)$ be the reduced coordinate ring of $\mathcal{V}_{P}$, and denote $q H_{\mathbb{C}}^{*}(X):=q H^{*}(X) \otimes \mathbb{C}$. The first main result of this paper is to give the following isomorphisms explicitly:

(1) $q H_{\mathbb{C}}^{*}\left(O G^{o}(n)\right) \stackrel{\sim}{\rightarrow} \mathcal{O}\left[\mathcal{V}_{n}^{C}\right]$,

(2) $q H_{\mathbb{C}}^{*}(L G(n)) \stackrel{\sim}{\rightarrow} \mathcal{O}\left[\mathcal{V}_{n}^{B}\right]$

(3) $q H_{\mathbb{C}}^{*}\left(O G^{e}(n)\right) \stackrel{\sim}{\rightarrow} \mathcal{O}\left[\mathcal{V}_{n+1}^{D}\right]$.

As a second result, we give Vafa-Intriligator type formulas to compute the Gromov-Witten invariants for $O G^{o}(n)\left(O G^{e}(n)\right)$ and $L G(n)$.

Let $\zeta=\zeta_{n}$ be the primitive $2 n$-th root of unity, i.e., $\zeta_{n}=e^{\frac{\pi i}{n}}$, and $\mathcal{T}_{n}$ the set of all $n$-tuples $J=\left(j_{1}, \ldots, j_{n}\right),-\frac{n-1}{2} \leq j_{1}<\cdots<j_{n} \leq \frac{3 n-1}{2}$, such that $\zeta^{J}:=\left(\zeta^{j_{1}}, \ldots, \zeta^{j_{n}}\right)$ is an $n$-tuple of distinct $2 n$-th roots of $(-1)^{n+1}$. Denote $\mathcal{I}_{n}:=$ $\left\{I \in \mathcal{T}_{n} \mid \zeta^{i_{k}} \neq-\zeta^{i_{l}}\right.$ for all $\left.k, l=1, \ldots, n\right\}$. For $J \in \mathcal{I}_{n}$, define $J^{*}$ to be an $n$-tuple 
$J^{*}=\left(j_{1}^{*}, \ldots, j_{n}^{*}\right) \in \mathcal{I}_{n}$ such that the two sets $\left\{\zeta^{j_{1}}, \ldots, \zeta^{j_{n}}\right\}$ and $\left\{\zeta^{n-j_{1}^{*}}, \ldots, \zeta^{n-j_{n}^{*}}\right\}$ enumerate all $2 n$-th roots of $(-1)^{n+1}$. Let $\widetilde{Q}_{\lambda}$ and $\widetilde{P}_{\lambda}$ be the $\widetilde{Q}$ - and $\widetilde{P}$-polynomials of Pragacz-Ratajski (see 2.5 or [18]), and let $P_{\lambda}$ be the Hall-Littlewood symmetric polynomial ([14]).

(1) Vafa-Intriligator type formula for $O G^{e}(n)\left(\cong O G^{o}(n)\right)$ :

Given Schubert classes $\tau_{\lambda}, \tau_{\mu}, \tau_{\nu}$ of $O G^{e}(n)$, and a nonnegative integer $k$, the Gromov-Witten invariants $\left\langle\tau_{\lambda}, \tau_{\mu}, \tau_{\hat{\nu}}\right\rangle_{k}$ are given by

$$
\begin{aligned}
& \left\langle\tau_{\lambda}, \tau_{\mu}, \tau_{\hat{\nu}}\right\rangle_{k} \\
& =\frac{2^{l(\nu)+2 k}}{(2 n)^{n}} \sum_{m=0}^{a(\nu)} \sum_{J \in \mathcal{I}_{n}} \widetilde{P}_{\lambda}\left(\zeta^{J}\right) \widetilde{P}_{\mu}\left(\zeta^{J}\right) P_{\nu(m)}\left(\zeta^{J^{*}}\right) \mid \text { Vand }\left.\left(\zeta^{J}\right)\right|^{2},
\end{aligned}
$$

whenever $|\lambda|+|\mu|=|\nu|+2 n k$, and otherwise by $\left\langle\tau_{\lambda}, \tau_{\mu}, \tau_{\hat{\nu}}\right\rangle_{k}=0$.

Here $a(\nu):=\left\lfloor\frac{n-l(\nu)}{2}\right\rfloor, \nu(m):=\left((n)^{(2 m)}, \nu_{1}, \ldots, \nu_{l}\right)$, and $\operatorname{Vand}\left(\zeta^{J}\right):=$ $\prod_{k<l}\left(\zeta^{j_{k}}-\zeta^{j_{l}}\right)$.

(2) Vafa-Intriligator type formula for $L G(n)$ :

Given Schubert classes $\sigma_{\lambda}, \sigma_{\mu}, \sigma_{\nu}$ of $L G(n)$, and a nonnegative integer $d$, the Gromov-Witten invariants $\left\langle\sigma_{\lambda}, \sigma_{\mu}, \sigma_{\hat{\nu}}\right\rangle_{d}$ are given by

$$
\begin{aligned}
& \left\langle\sigma_{\lambda}, \sigma_{\mu}, \sigma_{\hat{\nu}}\right\rangle_{d} \\
& =\frac{1}{2^{d}(2 n+2)^{(n+1)}} \sum_{m=0}^{b(\nu)} \sum_{J \in \mathcal{I}_{n+1}} \widetilde{Q}_{\lambda}\left(\zeta^{J}\right) \widetilde{Q}_{\mu}\left(\zeta^{J}\right) P_{\nu[m]}\left(\zeta^{J^{*}}\right)\left|\operatorname{Vand}\left(\zeta^{J}\right)\right|^{2},
\end{aligned}
$$

whenever $|\lambda|+|\mu|=|\nu|+(n+1) d$; otherwise $\left\langle\sigma_{\lambda}, \sigma_{\mu}, \sigma_{\hat{\nu}}\right\rangle_{d}=0$.

Here we denote $\zeta=\zeta_{n+1}$. For the notation of $b(\nu)$ and $\nu[m]$, see Proposition 7.5

Lastly we explicitly describe the totally nonnegative parts of the varieties $\mathcal{V}_{n}^{C}$ and $\mathcal{V}_{n}^{B}$ and show that they are isomorphic to $\mathbb{R}_{\geq 0}$. Furthermore for our cases we prove Rietsch's conjecture, which states that it is only on the totally nonnegative elements that the Schubert basis elements are nonnegative.

This paper is organized as follows. In Section 2, we collect some basic facts about Lie algebras in types $B, C$, and $D$, and give the definitions of $\widetilde{Q}$ - and $\widetilde{P}$-polynomials of Pragacz and Ratajski. In Section 3, we explain the quantum cohomology rings of Lagrangian and orthogonal Grassmannians and describe the result of Peterson. In Section 4, we give the Bruhat decomposition of the stabilizer $\left(U^{+}\right)^{e}$ of $e$ in $U^{+}$, determine by which regular functions the closure of a cell is defined, and state the main theorems on Peterson's result, Theorems 4.6, 4.8 and 4.10 and partially prove them. In Section 5 , we characterize elements of $\mathcal{V}_{n}^{B}, \mathcal{V}_{n}^{C}$ and $\mathcal{V}_{n+1}^{D}$ and describe how subvarieties of these varieties are positioned. In Section 6, we prove some orthogonality formulas for $\widetilde{Q}$ - and $\widetilde{P}$-polynomials at roots of unity and use these formulas to complete Theorems 4.6, 4.8 and 4.10. In Section 7 , we derive the VafaIntriligator type formulas for Lagrangian and orthogonal Grassmannians by using the formulas in Section 6, In Section 8, we give a quick review of total positivity of $U^{+}$and describe the totally positive parts of $\mathcal{V}_{n}^{B}$ and $\mathcal{V}_{n}^{C}$.

\section{Preliminaries}

2.1. Notation. Let $G$ be a complex semisimple algebraic group with rank $n$. Let $B=B^{-}$and $B^{+}$be opposite Borel subgroups, and $U^{-}$and $U^{+}$be the unipotent 
radicals of $B^{-}$and $B^{+}$, respectively. Let $T=B^{-} \cap B^{+}$be a maximal torus of $G$. Let $\mathfrak{g}, \mathfrak{b}^{-}, \mathfrak{b}^{+}, \mathfrak{u}^{-}, \mathfrak{u}^{+}$and $\mathfrak{t}$ be the Lie algebras of $G, B^{-}, B^{+}, U^{-}, U^{+}$, and $T$, respectively, so that the Lie algebra $\mathfrak{g}$ has the Cartan decomposition $\mathfrak{g}=\mathfrak{u}^{-} \oplus \mathfrak{t} \oplus \mathfrak{u}^{+}$. Let $e_{i}, h_{i}$ and $f_{i}, i=1, \ldots, n$, be the standard generators of $\mathfrak{g}$, and let $A=\left(a_{i j}\right)$ be the Cartan matrix of $\mathfrak{g}$.

We denote by $\triangle$ the root system for $\mathfrak{g}$ and by $\Delta^{+}$the set of positive roots in $\triangle$ and by $\Pi:=\left\{\alpha_{1}, \ldots, \alpha_{n}\right\} \subset \triangle$ the set of simple roots, which are defined by $\alpha_{j}\left(h_{i}\right)=a_{i j}$. Let $G^{\vee}$ be the Langlands dual of $G$, and denote the counterparts in $G^{\vee}$ of the above subgroups and their Lie algebras by the same alphabets as above with ${ }^{\vee}$. The $W$ eyl group $W$ of $G$ is defined as $W=\operatorname{Norm}_{G}(T) / T$. The action of $W$ on $\mathrm{T}$ induces the action of $W$ on $\mathfrak{t}$ and the dual action on $\mathfrak{t}^{*}$. If $W$ is generated by simple reflections $s_{i}, i \in I$, the action of $W$ on $\mathfrak{t}^{*}$ is given by $s_{i}(\gamma)=\gamma-\gamma\left(h_{i}\right) \alpha_{i}$, where $\gamma \in \mathfrak{t}^{*}, h_{i} \in \mathfrak{t}$, and $\alpha_{i} \in \Pi$. Note that the Weyl groups of $G$ and $G^{\vee}$ are identical to each other.

Let $w_{0}$ be the longest element of $W$, and for a parabolic subgroup $P$, let $W_{P}$ be the Weyl group of $P$ and $w_{P}$ the longest element of $W_{P}$. Then $w^{P}:=w_{0} w_{P}$ is the minimal length representative in the coset $w_{0} W_{P} \in W / W_{P}$. If a parabolic subgroup $P$ corresponds to a subset $J \subset I:=\{1, \ldots, n\}$, we write $P=P_{J}$, and we use alternatively $J$ and $P$ in the subscript or superscript of the above notation, e.g., $w^{J}=w^{P}$. If $P$ is a maximal parabolic subgroup corresponding to a fundamental weight $\kappa_{r}$ for some $r \in I$, i.e., $P=P_{J}$ for $J=I \backslash\{r\}$, then we write $P_{r}$ for $P_{J}$.

A fundamental weight $\kappa_{r}$ is minuscule if each weight of the fundamental representation $V_{r}$ corresponding to $\kappa_{r}$ is extremal, that is, if it is of the form $w \cdot \kappa_{r}$ for some $w \in W$. A maximal parabolic subgroup $P_{r}$ is called minuscule if the fundamental weight $\kappa_{r}$ is minuscule. We have the following list of the minuscule weights in classical Lie groups.

Type $A_{n}$ : Every fundamental weight is minuscule.

Type $B_{n}: \kappa_{n}$ is minuscule.

Type $C_{n}: \kappa_{1}$ is minuscule.

Type $D_{n}: \kappa_{1}, \kappa_{n-1}, \kappa_{n}$ are minuscule.

From the above list, we note that the parabolic subgroups $P_{n} \subset S O_{2 n+1}(\mathbb{C})$ and $P_{n+1} \subset S O_{2 n+2}(\mathbb{C})$ are minuscule, but the parabolic subgroup $P_{n} \subset S p_{2 n}(\mathbb{C})$ is not minuscule. We will mainly work with these three parabolic subgroups.

In the following three subsections, we summarize some basic facts about Lie algebras in types $B, C$ and $D$, which will be used in later sections. We will take a different ordered basis for $V$ than the usual Lie theory literature does, in order to embed the Lie groups in $B, C$ and $D$ into the Lie group in type $A$ more naturally. 2.2. $S p_{2 n}(\mathbb{C})$ and $\mathfrak{s p}_{2 n}(\mathbb{C})$. Let $V$ be a $2 n$-dimensional complex vector space equipped with a nondegenerate skew-symmetric bilinear form $Q$. Then the symplectic Lie group $S p_{2 n}(\mathbb{C})$ is defined to be the group of automorphisms $A$ of $V$ preserving $Q$, i.e., $Q(A v, A w)=Q(v, w)$ for all $v, w \in V$. The Lie algebra $\mathfrak{s p}_{2 n}(\mathbb{C})$ of $S p_{2 n}(\mathbb{C})$ is the vector space of endomorphisms $X: V \rightarrow V$ such that $Q(X v, w)+Q(v, X w)=0$ for all $v, w \in V$. Choose an ordered basis $\xi_{1}, \ldots, \xi_{2 n}$ for $V$ such that

$$
Q\left(\xi_{i}, \xi_{i^{*}}\right)=-Q\left(\xi_{i^{*}}, \xi_{i}\right)=(-1)^{i+1}
$$

and

$$
Q\left(\xi_{i}, \xi_{j}\right)=0 \text { if } i \neq j^{*},
$$


where $i^{*}:=2 n+1-i$. Let $J$ be the $2 n \times 2 n$ matrix defined by

$$
J_{i, j}:=(-1)^{i+1} \delta_{i, j^{*}}, \quad i=1, \ldots, 2 n .
$$

Then $S p_{2 n}(\mathbb{C})$ is the group of $2 n \times 2 n$ matrices $A$ with $J=A^{t} \cdot J \cdot A$, and $\mathfrak{s p}_{2 n}(\mathbb{C})$ is the space of $2 n \times 2 n$ matrices $X$ with $X^{t} \cdot J+J \cdot X=0$.

For positive integers $i, j$, let $E_{i, j}$ be the $2 n \times 2 n$ matrices such that the $(i, j)$-th entry is 1 , and 0 elsewhere, and let $\bar{h}_{i}:=E_{i, i}-E_{i^{*}, i^{*}}$. As a Cartan subalgebra $\mathfrak{t}$ for $\mathfrak{s p}_{2 n}(\mathbb{C})$, we take the subalgebra generated by $\bar{h}_{i}, i=1, \ldots, n$. Let $l_{1}, \ldots, l_{n}$ be a basis of $\mathfrak{t}^{*}$ dual to $\bar{h}_{1}, \ldots, \bar{h}_{n}$. The set $\triangle=\left\{ \pm l_{i} \pm l_{j} \mid 1 \leq i, j \leq n\right\}$ forms a root system for $\mathfrak{s p}_{2 n}(\mathbb{C})$. The set $\Pi$ of simple roots consists of $\alpha_{i}:=l_{i}-l_{i+1}$ for $i=1, \ldots, n-1$, and $\alpha_{n}:=2 l_{n}$, and the simple root vectors $([2])$ are

$$
\begin{aligned}
e_{i} & =E_{i, i+1}+E_{i^{*}-1, i^{*}} \quad \text { for } i=1, \ldots, n-1, \\
e_{n} & =E_{n, n+1} .
\end{aligned}
$$

The fundamental dominant weights corresponding to $\alpha_{i}$ are $\kappa_{i}=l_{1}+\cdots+l_{i}, i=$ $1, \ldots, n$, and the fundamental representation $V_{i}$ corresponding to $\kappa_{i}$ is the subspace of $\wedge^{i} V$ generated by the highest weight vector $\xi_{1} \wedge \cdots \wedge \xi_{i}$.

The Weyl group $W_{n}$ for the root system $C_{n}$ is an extension of the symmetric group $S_{n}=\left\langle s_{1}, \ldots, s_{n-1}\right\rangle$ by $\dot{s}_{n}$, which acts on the right; in the notation of bar permutations,

$$
\left(u_{1}, \ldots, u_{n}\right) \dot{s}_{n}=\left(u_{1}, \ldots, u_{n-1}, \bar{u}_{n}\right) .
$$

The generators $s_{1}, \ldots, s_{n-1}$, and $\dot{s}_{n}$ act naturally on $\mathrm{t}^{*}$; for $i \leq n-1, s_{i}$ interchanges $l_{i}$ and $l_{i+1}$, and $\dot{s}_{n}$ interchanges $\pm l_{n}$ and $\mp l_{n}$. Note that the maximal length element $w_{0}$ in $W_{n}$ is $(\overline{1}, \overline{2}, \ldots, \bar{n})$.

2.3. $S O_{2 n+1}(\mathbb{C})$ and $\mathfrak{s o}_{2 n+1}(\mathbb{C})$. Let $V$ be a $(2 n+1)$-dimensional complex vector space equipped with a nondegenerate symmetric bilinear form $Q: V \times V \rightarrow \mathbb{C}$. The orthogonal group $S O_{2 n+1}(\mathbb{C})$ is defined to be the group of automorphisms $A$ of $V$ of determinant 1 preserving $Q$; that is, $Q(A v, A w)=Q(v, w)$ for all $v, w \in V$, and its Lie algebra $\mathfrak{s o}_{2 n+1}(\mathbb{C})$ is a vector space of endomorphisms $X: V \rightarrow V$ such that $Q(X v, w)+Q(v, X w)=0$ for all $v, w \in V$. Let $\xi_{1}, \ldots, \xi_{2 n+1}$ be a basis for $V$ such that

$$
Q\left(\xi_{i}, \xi_{i^{*}}\right)=Q\left(\xi_{i^{*}}, \xi_{i}\right)=(-1)^{i+1}
$$

and

$$
Q\left(\xi_{i}, \xi_{j}\right)=0 \text { if } j \neq i^{*},
$$

where $i^{*}:=2 n+2-i$. Let $J$ be the $(2 n+1) \times(2 n+1)$ matrix defined by

$$
J_{i, j}:=(-1)^{i+1} \delta_{i, j^{*}}, \quad i, j=1, \ldots, 2 n+1 .
$$

Then $S O_{2 n+1}(\mathbb{C})$ is the group of matrices $A$ satisfying the relations $J=A^{t} \cdot J \cdot A$, and the Lie algebra $\mathfrak{s o}_{2 n+1}(\mathbb{C})$ is the space of matrices $X$ satisfying the relation $X^{t} \cdot J+J \cdot X=0$. Define $E_{i, j}, l_{i}$ and $\bar{h}_{i}$ as in $\$ 2.2$. As a Cartan subalgebra we take the subalgebra generated by $\bar{h}_{i}, i=1, \ldots, n$. The set $\triangle=\left\{ \pm l_{i} \pm l_{j} \mid i, j=\right.$ $1, \ldots, n$, and $i \neq j\} \cup\left\{ \pm l_{i} \mid i=1, \ldots, n\right\}$ forms a root system for $\mathfrak{s o}_{2 n+1}(\mathbb{C})$. The set of simple roots consists of $\alpha_{i}:=l_{i}-l_{i+1}$ for $i=1, \ldots, n-1$, and $\alpha_{n}:=l_{n}$. The simple root vectors $([2])$ are

$$
\begin{aligned}
e_{i} & =E_{i, i+1}+E_{i^{*}-1, i^{*}} \text { for } i=1, \ldots, n-1, \\
e_{n} & =\sqrt{2}\left(E_{n, n+1}+E_{n+1, n+2}\right) .
\end{aligned}
$$


The fundamental dominant weights are $\kappa_{i}=l_{1}+\cdots+l_{i}$ for $i=1, \ldots, n-1$, and $\kappa_{n}=\frac{1}{2}\left(l_{1}+\cdots+l_{n}\right)$. To each $\kappa_{i}, i=1, \ldots, n-1$, there corresponds a fundamental representation $V_{i}$ of $\mathfrak{s o}_{2 n+1}(\mathbb{C})$ which is the subspace of $\bigwedge^{i} V$ generated by the highest weight vector $\xi_{1} \wedge \cdots \wedge \xi_{i}$, and to $\kappa_{n}$ the spin representation $V_{n}^{s}([4)$. The group $S O_{2 n+1}(\mathbb{C})$ has $V_{1}, \ldots, V_{n-1}$ as fundamental representations, but not $V_{n}^{s}$. But there is a representation $V_{n}$ which corresponds to the weight $l_{1}+\cdots+l_{n}$. This is the subspace of $\bigwedge^{n} V$ generated by the highest weight vector $\xi_{1} \wedge \cdots \wedge \xi_{n}$. We consider $V_{n}$ as a fundamental representation as well.

As a matter of convenience for later use, we give basic facts on $S_{2 n+2}(\mathbb{C})$ and $\mathfrak{s o}_{2 n+2}(\mathbb{C})$ rather than $S O_{2 n}(\mathbb{C})$ and $\mathfrak{s o}_{2 n}(\mathbb{C})$.

2.4. $S O_{2 n+2}(\mathbb{C})$ and $\mathfrak{s o}_{2 n+2}(\mathbb{C})$. Let $V$ be a $(2 n+2)$-dimensional vector space with a nondegenerate bilinear form $Q$. The definitions of $S_{2 n+2}(\mathbb{C})$ and $\mathfrak{s o}_{2 n+2}(\mathbb{C})$ are the same as those of $S O_{2 n+1}(\mathbb{C})$ and $\mathfrak{s o}_{2 n+1}(\mathbb{C})$. We take an ordered basis $\xi_{1}, \ldots, \xi_{2 n+2}$ for $V$ such that

$$
Q\left(\xi_{i}, \xi_{i^{*}}\right)=Q\left(\xi_{i^{*}}, \xi_{i}\right)=1
$$

and

$$
Q\left(\xi_{i}, \xi_{j}\right)=0 \text { if } j \neq i^{*},
$$

where $i^{*}:=2 n+3-i$. Let $E_{i, j}, \bar{h}_{i}$ and $l_{i}$ be defined as in $₫ 2.2$. As a Cartan subalgebra we take the subalgebra generated by $\bar{h}_{i}, i=1, \ldots, n+1$. The set of roots of $\mathfrak{s o}_{2 n+2}(\mathbb{C})$ is

$$
\triangle=\left\{ \pm l_{i} \pm l_{j} \mid i, j=1, \ldots, n+1, i \neq j\right\}
$$

and the set $\Pi$ of simple roots consists of $\alpha_{i}=l_{i}-l_{i+1}, i=1, \ldots, n$, and $\alpha_{n+1}=$ $l_{n}+l_{n+1}$. The corresponding simple root vectors are

$$
\begin{aligned}
e_{i} & =E_{i, i+1}-E_{i^{*}-1, i^{*}} \text { for } i=1, \ldots, n, \\
e_{n+1} & =E_{n, n+2}-E_{n+1, n+3} .
\end{aligned}
$$

Denote $\omega_{i}:=l_{1}+\cdots+l_{i}$ for $i=1, \ldots, n$, and $\omega_{n+1}^{ \pm}:=l_{1}+\cdots+l_{n} \pm l_{n+1}$. Then the fundamental dominant weights $\kappa_{i}$ corresponding to $\alpha_{i}$ are as follows: $\kappa_{i}=\omega_{i}$ for $i=1, \ldots, n-1, \kappa_{n}=\frac{1}{2} \omega_{n+1}^{-}$, and $\kappa_{n+1}=\frac{1}{2} \omega_{n+1}^{+}$. To a fundamental weight $\kappa_{i}, i=$ $1, \ldots, n-1$, there corresponds the fundamental representation $V_{i}$ of $\mathfrak{s o}_{2 n+2}(\mathbb{C})$, which is the subspace of $\bigwedge^{i} V$ generated by the highest weight vector $\xi_{1} \wedge \cdots \wedge \xi_{i}$, and to the fundamental weights $\kappa_{n}$ and $\kappa_{n+1}$ there correspond the spin representations $V_{n}^{s}$ and $V_{n+1}^{s}$, respectively ([4]). As fundamental representations, the group $S O_{2 n+2}(\mathbb{C})$ has $V_{i}, i=1, \ldots, n-1$, but not the spin representations $V_{n}^{s}$ and $V_{n+1}^{s}$. But there are representations $V_{n}$ and $V_{n+1}^{ \pm}$of $S O_{2 n+2}(\mathbb{C})$, which correspond to the weights $\omega_{n}$ and $\omega_{n+1}^{ \pm}$, respectively. The representation $V_{n}$ is the subspace of $\bigwedge^{n} V$ generated by the highest weight vector $\xi_{1} \wedge \cdots \wedge \xi_{n}$, and $V_{n+1}^{ \pm}$are the subspaces of $\wedge^{n+1} V$ generated by the highest weight vectors $\xi_{1} \wedge \cdots \wedge \xi_{n+1}$ for $(+) \operatorname{sign}$ and $\xi_{1} \wedge \cdots \wedge \xi_{n} \wedge \xi_{n+2}$ for $(-)$ sign. Note that these representations generate all the representations of $S O_{2 n+2}(\mathbb{C})$, with the following relation (p. 379 in 4 ):

(2.2) $\left(V_{n+1}^{+} \oplus V_{n-1} \oplus V_{n-3} \oplus \cdots\right) \otimes\left(V_{n+1}^{-} \oplus V_{n-1} \oplus V_{n-3} \oplus \cdots\right)=\left(V_{n} \oplus V_{n-2} \oplus \cdots\right)^{2}$.

We will refer to these $(n+2)$ representations as fundamental ones of $S_{2 n+2}(\mathbb{C})$.

The Weyl group $\widetilde{W}_{n+1}$ for type $D_{n+1}$ is an extension of the symmetric group $S_{n+1}=\left\langle s_{1}, \ldots, s_{n}\right\rangle$ by an element $\tilde{s}_{n+1}$ which acts on the right by

$$
\left(u_{1}, \ldots, u_{n+1}\right) \tilde{s}_{n+1}=\left(u_{1}, \ldots, u_{n-1}, \bar{u}_{n+1}, \bar{u}_{n}\right) .
$$


Note that the maximal length element $\widetilde{w}_{0}$ of $\widetilde{W}_{n+1}$ is given by

$$
\widetilde{w}_{0}= \begin{cases}(\overline{1}, \ldots, \overline{n+1}) & \text { if } n \text { is odd } \\ (\overline{1}, \ldots, \bar{n}, n+1) & \text { if } n \text { is even }\end{cases}
$$

The action of $\widetilde{W}_{n+1}$ on $\mathfrak{t}^{*}$ is given as follows: for $i=1, \ldots, n, s_{i}$ interchanges $l_{i}$ and $l_{i+1}$, and $\tilde{s}_{n+1}$ interchanges $\pm l_{n}$ and $\mp l_{n+1}$.

2.5. Symmetric polynomials. A partition $\lambda$ is a weakly decreasing sequence $\lambda=\left(\lambda_{1}, \lambda_{2}, \ldots, \lambda_{m}\right)$ of nonnegative integers. A Young diagram is a collection of boxes, arranged in left-justified rows, with a weakly decreasing number of boxes in each row. To a partition $\lambda=\left(\lambda_{1}, \ldots, \lambda_{m}\right)$, we associate a Young diagram whose $i$-th row has $\lambda_{i}$ boxes. The nonzero $\lambda_{i}$ in $\lambda=\left(\lambda_{1}, \ldots, \lambda_{m}\right)$ are called the parts of $\lambda$. The number of parts of $\lambda$ is called the length of $\lambda$, denoted by $l(\lambda)$; the sum of the parts of $\lambda$ is called the weight of $\lambda$, denoted by $|\lambda|$. For positive integers $m$ and $n$, denote by $\mathcal{R}(m, n)$ the set of all partitions whose Young diagram fits inside an $m \times n$ diagram, which is the Young diagram of the partition $\left(n^{m}\right)$. A partition $\lambda=\left(\lambda_{1}, \ldots, \lambda_{m}\right) \in \mathcal{R}(m, n)$ is called strict if $\lambda_{1}>\cdots>\lambda_{m}$. Denote by $\mathcal{D}(m, n)$ the set of all strict partitions in $\mathcal{R}(m, n)$. If $m=n$, then we write $\mathcal{R}(n)$ and $\mathcal{D}(n)$ for $\mathcal{R}(n, n)$ and $\mathcal{D}(n, n)$, respectively. If $\lambda \in \mathcal{D}(n)$, denote by $\widehat{\lambda}$ the partition whose parts complement the parts of $\lambda$ in the set $\{1, \ldots, n\}$. For $\lambda \in \mathcal{R}(m, n)$, the conjugate of $\lambda$ is the partition $\lambda^{t} \in \mathcal{R}(n, m)$ whose Young diagram is the transpose of that of $\lambda$. We define $\widetilde{Q}$ - and $\widetilde{P}$-polynomials of Pragacz and Ratajski as follows. For $X_{n}:=\left(x_{1}, \ldots, x_{n}\right)$, set $\widetilde{Q}_{i}\left(X_{n}\right):=E_{i}\left(X_{n}\right)$, the $i$-th elementary symmetric function. Given two nonnegative integers $i$ and $j$ with $i \geq j$, define

$$
\widetilde{Q}_{i, j}\left(X_{n}\right)=\widetilde{Q}_{i}\left(X_{n}\right) \widetilde{Q}_{j}\left(X_{n}\right)+2 \sum_{k=1}^{j}(-1)^{k} \widetilde{Q}_{i+k}\left(X_{n}\right) \widetilde{Q}_{j-k}\left(X_{n}\right) .
$$

Finally, for any partition $\lambda$ of length $l=l(\lambda)$, not necessarily strict, and for $r=2\lfloor(l+1) / 2\rfloor$, let $B_{\lambda}^{r}=\left[B_{i, j}\right]_{1 \leq i, j \leq r}$ be the skew symmetric matrix defined by $B_{i, j}=\widetilde{Q}_{\lambda_{i}, \lambda_{j}}\left(X_{n}\right)$ for $i<j$. We set

$$
\widetilde{Q}_{\lambda}\left(X_{n}\right)=\operatorname{Pfaffian}\left(B_{\lambda}^{r}\right) .
$$

The $\widetilde{Q}$-polynomials satisfy the following properties, which will be used in later sections.

(1) $\widetilde{Q}_{i, i}\left(X_{n}\right)=E_{i}\left(x_{1}^{2}, \ldots, x_{n}^{2}\right)$.

(2) For partitions $\lambda=\left(\lambda_{1}, \ldots, \lambda_{l}\right)$ and $\lambda^{\prime}=\left(\lambda_{1}, \lambda_{2}, \ldots, j, j, \ldots, \lambda_{l}\right)=\lambda \cup(j, j)$,

$$
\widetilde{Q}_{\lambda^{\prime}}\left(X_{n}\right)=\widetilde{Q}_{j, j}\left(X_{n}\right) \widetilde{Q}_{\lambda}\left(X_{n}\right) .
$$

(3) For any $\lambda \in \mathcal{D}(n)$,

$$
\widetilde{Q}_{\lambda}\left(X_{n}\right) \widetilde{Q}_{n}\left(X_{n}\right)=\widetilde{Q}_{\left(n, \lambda_{1}, \ldots, \lambda_{l}\right)}\left(X_{n}\right) .
$$

Given $\lambda$, not necessarily strict, $\widetilde{P}_{\lambda}$ is defined by

$$
\widetilde{P}_{\lambda}\left(X_{n}\right):=2^{-l(\lambda)} \widetilde{Q}_{\lambda}\left(X_{n}\right) .
$$


Note that $\widetilde{P}$-polynomials enjoy the factorization properties (2) and (3) of $\widetilde{Q}$. For $i=1, \ldots, n$, let $H_{i}\left(X_{n}\right)$ be the $i$-th complete symmetric function. Then for any partition $\lambda$, the Schur polynomial $S_{\lambda}\left(X_{n}\right)$ is defined by

$$
S_{\lambda}\left(X_{n}\right):=\operatorname{Det}\left[H_{\lambda_{i}-j+i}\left(X_{n}\right)\right]_{1 \leq i, j \leq n}=\operatorname{Det}\left[E_{\lambda_{i}^{t}-j+i}\left(X_{n}\right)\right]_{1 \leq i, j \leq n},
$$

where $H_{0}\left(X_{n}\right)=E_{0}\left(X_{n}\right)=1$ and $H_{k}\left(X_{n}\right)=E_{k}\left(X_{n}\right)=0$ for $k<0$. See [18] for a further reference on $\widetilde{Q}$ - and $\widetilde{P}$-polynomials, and $[3$ or $[14$ for the Schur polynomials.

\section{Quantum Cohomology Rings And Peterson's Result}

The quantum cohomology ring of a projective manifold $X$ is defined via 3-point genus zero Gromov-Witten invariants. Informally speaking, these Gromov-Witten invariants are defined originally as intersection numbers on the moduli space of certain stable maps to $X$. But if $X$ is convex, e.g., $X=G / P$, this original definition is enumerative; that is, the Gromov-Witten invariant counts the numbers of rational maps $f: \mathbb{P}^{1} \rightarrow X$ satisfying certain conditions. For the sake of simplicity, as a definition of Gromov-Witten invariants we take this enumerative one rather than the original one. We refer to 5 for the original definition. In this section, using the Gromov-Witten invariants so defined, we introduce the quantum cohomology rings of $L G(n)$ and $O G^{e}(n)$, give presentations of these rings, and describe Peterson's result on the quantum cohomology ring of $G / P$.

3.1. Quantum cohomology ring of Lagrangian Grassmannian. To describe the quantum cohomology of $L G(n)$, we define the Schubert varieties indexed by the strict partitions in $\mathcal{D}(n)$. Let $V$ be a complex vector space of dimension $2 n$ with a nondegenerate skew-symmetric form. Let $F$. be a fixed complete isotropic flag of subspaces $F_{i}$ of $V$ :

$$
F .: 0=F_{0} \subset F_{1} \subset \cdots \subset F_{n} \subset V
$$

where $\operatorname{dim}\left(F_{i}\right)=i$ for each $i$, and $F_{n}$ is Lagrangian. Define the Schubert variety $X_{\lambda}(F$. $)$ as the locus of $\Sigma \in L G(n)$ such that

$$
\operatorname{dim}\left(\Sigma \cap F_{n+1-\lambda_{i}}\right) \geq i \text { for } i=1, \ldots, l(\lambda) .
$$

Then $X_{\lambda}\left(F\right.$.) is a subvariety of $L G(n)$ of complex codimension $|\lambda|$. Let $\sigma_{\lambda}$ be the class of $X_{\lambda}(F$. $)$ in the cohomology group $H^{2|\lambda|}(L G(n))$. Then $\sigma_{\lambda}$ with $\lambda \in \mathcal{D}(n)$ forms an additive basis for $H^{*}(L G(n))$.

A rational map of degree $d$ to $L G(n)$ is a morphism $f: \mathbb{P}^{1} \rightarrow L G(n)$ such that

$$
\int_{L G(n)} f_{*}\left[\mathbb{P}^{1}\right] \cdot \sigma_{1}=d
$$

Given an integer $d \geq 0$ and partitions $\lambda, \mu, \nu \in \mathcal{D}(n)$ with $|\lambda|+|\mu|+|\nu|=$ $\operatorname{dim}(L G(n))+d(n+1)$, the Gromov-Witten invariants $\left\langle\sigma_{\lambda}, \sigma_{\mu}, \sigma_{\nu}\right\rangle_{d}$ are defined as the number of rational maps $f: \mathbb{P} \rightarrow L G(n)$ such that $f(0) \in X_{\lambda}(F$. $), f(1) \in$ $X_{\mu}\left(G\right.$.), and $f(\infty) \in X_{\nu}\left(H\right.$.), for given isotropic flags $F$., $G_{\text {. }}$, and $H$. in general position. The quantum cohomology ring $q H^{*}(L G(n))$ is isomorphic to $H^{*}(L G(n)) \otimes_{\mathbb{Z}}$ $\mathbb{Z}[q]$ as a $\mathbb{Z}[q]$-module, where $q$ is a formal variable of degree $(n+1)$. The multiplication in $q H^{*}(L G(n))$ is given by the relation

$$
\sigma_{\lambda} \cdot \sigma_{\mu}=\sum\left\langle\sigma_{\lambda}, \sigma_{\mu}, \sigma_{\hat{\nu}}\right\rangle_{d} \sigma_{\nu} q^{d}
$$


where the sum is taken over $d \geq 0$ and partitions $\nu$ with $|\nu|=|\lambda|+|\mu|-(n+1) d$. We have the following presentation of the quantum cohomology ring of $L G(n)$, due to Kresch and Tamvakis.

Theorem $3.1([9])$. The ring $q H^{*}(L G(n))$ is presented as a quotient of the polynomial ring $\mathbb{Z}\left[\sigma_{1}, \ldots, \sigma_{n}, q\right]$ by the relations

$$
\sigma_{r}^{2}+2 \sum_{i=1}^{n-r}(-1)^{i} \sigma_{r+i} \sigma_{r-i}=(-1)^{n-r} \sigma_{2 r-n-1} q
$$

for $1 \leq r \leq n$.

See [9] for more details on the quantum cohomology ring of $L G(n)$.

3.2. Quantum cohomology ring of orthogonal Grassmannian. Let $V$ be a complex vector space of dimension $2 n+2$ with a nondegenerate symmetric form. The Schubert varieties $X_{\lambda}(F$. $)$ are parametrized by partitions $\lambda \in \mathcal{D}(n)$, and are defined by the same equation (3.4) as before, relative to an isotropic flag $F$. in $V$. Let $\tau_{\lambda}$ be the cohomology class of $X_{\lambda}\left(F\right.$. . Then the $\tau_{\lambda}$ for $\lambda \in \mathcal{D}(n)$ form a $\mathbb{Z}$-basis for $H^{*}\left(O G^{e}(n)\right)$. The cohomology ring $H^{*}\left(O G^{e}(n)\right)$ can be presented in terms of $\widetilde{P}$ - polynomials. More precisely, let $\Lambda_{n}$ denote the $\mathbb{Z}$-algebra generated by the polynomials $\widetilde{P}_{i}\left(X_{n}\right)$ for all $i=1, \ldots, n$. Then the map from $\Lambda_{n}$ to $H^{*}\left(O G^{e}(n)\right)$ sending $\widetilde{P}_{\lambda}\left(X_{n}\right)$ to $\tau_{\lambda}$ is a surjective ring homomorphism with the kernel generated by the polynomials $\widetilde{P}_{i, i}\left(X_{n}\right)$ for all $i=1, \ldots, n([10])$.

In this case, the Gromov-Witten invariants $\left\langle\tau_{\lambda}, \tau_{\mu}, \tau_{\nu}\right\rangle_{k}$ are defined for $|\lambda|+|\mu|+$ $|\nu|=\operatorname{deg}\left(O G^{e}(n)\right)+2 n k$ and count the number of rational maps $f: \mathbb{P}^{1} \rightarrow O G^{e}(n)$ of degree $k$ such that $f(0) \in X_{\lambda}(F),. f(1) \in X_{\mu}(G$. $)$, and $f(\infty) \in X_{\nu}\left(H_{\text {. }}\right)$, for given isotropic flags $F$. $G$., and $H$. in general position. The quantum cohomology ring of $O G^{e}(n)$ is isomorphic to $H^{*}\left(O G^{e}(n)\right) \otimes \mathbb{Z}[q]$ as a $\mathbb{Z}[q]$-module. The multiplication in $q H^{*}\left(O G^{e}(n)\right)$ is given by the relation

$$
\tau_{\lambda} \cdot \tau_{\mu}=\sum\left\langle\tau_{\lambda}, \tau_{\mu}, \tau_{\hat{\nu}}\right\rangle_{k} \tau_{\nu} q^{k}
$$

where the sum is taken over $k \geq 0$ and partitions $\nu$ with $|\nu|=|\lambda|+|\mu|-2 n k$.

Theorem 3.2 ([10]). The quantum cohomology ring $q H^{*}\left(O G^{e}(n)\right)$ is presented as a quotient of the polynomial ring $\mathbb{Z}\left[\tau_{1}, \ldots, \tau_{n}, q\right]$ modulo the relations $\tau_{r, r}=0$ for $r=1, \ldots, n-1$ together with the quantum relation $\tau_{n}^{2}=q$, where

$$
\tau_{r, r}:=\tau_{r}^{2}+2 \sum_{i=1}^{r-1}(-1)^{i} \tau_{r+i} \tau_{r-i}+(-1)^{r} \tau_{2 r} .
$$

See [10] for more details on the quantum cohomology of $O G^{e}(n)$ and $O G^{o}(n)$.

3.3. Peterson's results for the quantum cohomology $\operatorname{ring}$ of $G / P$. For $i \in I$, let $e_{i}^{\vee}$ be weight vectors of simple roots for $\mathfrak{g}^{\vee},\left(e_{i}^{\vee}\right)^{*}$ the linear functional which is one on $e_{i}^{\vee}$ and zero on the weight vectors of all the other simple roots, and set

$$
e^{\vee}:=\sum_{i \in I} e_{i}^{\vee} \in \mathfrak{g}^{\vee} \text { and }\left(e^{\vee}\right)^{*}:=\sum_{i \in I}\left(e_{i}^{\vee}\right)^{*} \in\left(\mathfrak{g}^{\vee}\right)^{*} .
$$

For $g \in G^{\vee}$, let $A d_{g}^{*}$ denote the coadjoint action of $g$ on $\left(\mathfrak{g}^{\vee}\right)^{*}$, and let $\left(\left(U^{\vee}\right)^{+}\right)^{e^{\vee}}$ be the stabilizer of $e^{\vee}$ in $\left(U^{\vee}\right)^{+}$. Then the Peterson variety is defined as

$$
\mathcal{Y}:=\left\{g\left(B^{-}\right)^{\vee} \in G^{\vee} /\left(B^{\vee}\right)^{-} \mid A d_{g}^{*}\left(e^{\vee}\right)^{*} \text { vanishes on }\left[\left(\mathfrak{u}^{\vee}\right)^{-},\left(\mathfrak{u}^{\vee}\right)^{-}\right]\right\} \text {. }
$$


For a parabolic subgroup $P \subseteq G$, define $\mathcal{Y}_{P}$ as

$$
\mathcal{Y}_{P}:=\mathcal{Y} \times_{G^{\vee} / B^{\vee}}\left[\left(B^{\vee}\right)^{+} w_{P}\left(B^{\vee}\right)^{-} /\left(B^{\vee}\right)^{-}\right] \text {(scheme-theoretic intersection). }
$$

Then the subvarieties $\mathcal{Y}_{P}$ form strata of the variety $\mathcal{Y}$, i.e,

$$
\mathcal{Y}=\bigsqcup_{P} \mathcal{Y}_{P}
$$

where $P$ runs over the set of all parabolic subgroups $P$ of $G$ containing $B$, and it is known that the variety $\mathcal{Y}_{P}$ need not be reduced, but is a local complete intersection. One of the key results of Peterson's may be stated as follows:

The quantum cohomology ring $q H_{\mathbb{C}}^{*}(G / P)$ of a homogeneous space $G / P$ is isomorphic to the coordinate ring of $\mathcal{Y}_{P}$.

If $P$ is a minuscule parabolic subgroup of $G$, Peterson's result goes further. For a parabolic subgroup $P$ of $G$, not necessarily minuscule, define

$$
\mathcal{V}_{P}:=\left(\left(U^{\vee}\right)^{+}\right)^{e^{\vee}} \cap \overline{\left(B^{\vee}\right)^{-} w^{P}\left(B^{\vee}\right)^{-}} .
$$

If $P$ is minuscule, the quantum cohomology ring $q H_{\mathbb{C}}^{*}(G / P)$ of a homogeneous space $G / P$ is isomorphic to the coordinate ring of $\mathcal{V}_{P}$. ([17]).

This follows from an isomorphism of two varieties $\mathcal{V}_{P} \stackrel{\sim}{\rightarrow} \mathcal{Y}_{P}, u \mapsto u w_{P}\left(B^{\vee}\right)^{-}$

This result was verified in an elementary way by Rietsch for minuscule parabolic subgroups and general parabolic subgroups of $S L_{N}(\mathbb{C})$ in [19] and [20], respectively. See [16] and 21] for more details on Peterson's results.

\section{Comparing two PRESEntations of QuANTUm COHOMOlOgY RingS}

In this section we will show that two presentations for $O G^{o}(n), O G^{e}(n)$ and $L G(n)$ by A. Kresch and H. Tamvakis and by D. Peterson are equivalent to each other. This provides an independent proof of Peterson's results for Lagrangian and orthogonal Grassmannians.

4.1. $\left(U^{+}\right)^{e}$ and its intersection with Bruhat cells. Throughout this paper, we fix the principal nilpotent element $e:=\sum_{i \in I} e_{i} \in \mathfrak{g}$. Let $\left(U^{+}\right)^{e}:=\left\{u \in\left(U^{+}\right)^{e} \mid\right.$ $\left.u e u^{-1}=e\right\}$, the stabilizer of $e$ in $U^{+}$. This is an abelian subgroup of $U^{+}$of dimension equal to the rank of $G(7)$.

Lemma 4.1 ([19]). The elements $w_{J}$ can be characterized by $\left\{w_{J} \in W \mid J \subseteq I\right\}=$ $\left\{w \in W \mid w \cdot \Pi \subseteq(-\Pi) \cup \triangle^{+}\right\}$.

Proof. For the proof, we refer to Lemma 2.2 in [19].

Lemma 4.2 ([19]). The Bruhat decomposition induces $\left(U^{+}\right)^{e}=\bigsqcup_{J \subseteq I}\left(U^{+}\right)^{e} \cap$ $B^{-} w^{J} B^{-}$.

Proof. If $u \in\left(U^{+}\right)^{e}$, then $u \in B^{-} w_{0} w B^{-}$for some $w \in W$. Write $u=b_{1} w_{0} w b_{2}$ for some $b_{1} \in B^{-}$and $b_{2} \in U^{-}$. Since $u e u^{-1}=e$, i.e., $u \cdot e=e$, we have

$$
w b_{2} \cdot e=w_{0} b_{1}^{-1} \cdot e .
$$

But note that $b_{2} \cdot e=e+x$ for some $x \in \mathfrak{b}^{-}$, and $w_{0} b_{1}^{-1} \cdot e=\sum_{i=1}^{n} a_{i} f_{i}+y$ for some $a_{i} \in \mathbb{C}$ and $y \in \mathfrak{b}^{+}$. From the equality

$$
w b_{2} \cdot e=\sum_{i=1}^{n} a_{i} f_{i}+y,
$$


we have $w \cdot \Pi \subset(-\Pi) \cup \triangle^{+}$. It follows from Lemma 4.1 that we have $w=w_{J}$ for some $J \subseteq I$.

Definition. Fix a dominant weight $\kappa$ of $\mathfrak{g}$, let $u \in\left(U^{+}\right)^{e}$, and define a regular function $\triangle_{\kappa}$ on $\left(U^{+}\right)^{e}$ as

$$
\triangle_{\kappa}(u)=\left\langle u \cdot v_{w_{0} \cdot \kappa}, v_{\kappa}\right\rangle,
$$

where $v_{\kappa}$ and $v_{w_{0} \cdot \kappa}$ are the highest and lowest weight vectors in the representation $V_{\kappa}$, respectively, and $\left\langle u \cdot v_{w_{0} \cdot \kappa}, v_{\kappa}\right\rangle$ is the coefficient of $v_{\kappa}$ in the decomposition on the weight spaces of $V_{\kappa}$ of $u \cdot v_{w_{0} \cdot \kappa}$.

Note that this definition makes sense because the highest and lowest weight spaces are one dimensional, and also note that the function $\triangle_{\kappa}$ is only defined up to a choice of highest and lowest weight vectors. The following two lemmas and corollary are the generalizations of ideas in Lemma 2.3 of [19].

Lemma 4.3. Let $u \in\left(U^{+}\right)^{e} \cap B^{-} w^{J} B^{-}$for some $J \subset I$, and let $\kappa$ be a dominant weight of $\mathfrak{g}$. Then $\triangle_{\kappa}(u) \neq 0$ precisely when $w_{0} w_{J} w_{0} \cdot \kappa=\kappa$.

Proof. Choose representatives for $w_{0}$ and $w_{J}$ from $\operatorname{Norm}_{G}(T)$, for which we also will write $w_{0}$ and $w_{J}$, and write $u=b_{1} w_{0} w_{J} b_{2}$ for some $b_{1} \in B^{-}, b_{2} \in U^{-}$. Then we have, up to nonzero scalar multiplication,

$$
u \cdot v_{w_{0} \cdot \kappa}=\left(b_{1} w_{0} w_{J} b_{2}\right) \cdot v_{w_{0} \cdot \kappa}=\left(b_{1} w_{0} w_{J}\right) \cdot v_{w_{0} \cdot \kappa}=b_{1} w_{0} w_{J} w_{0} \cdot v_{\kappa} .
$$

To prove the lemma, it suffices to show that $\left\langle u \cdot v_{w_{0} \cdot \kappa}, v_{\kappa}\right\rangle \neq 0$ precisely if $w_{0} w_{J} w_{0} \cdot v_{\kappa}$ $=v_{\kappa}$ up to nonzero scalar multiplication. Indeed if $w_{0} w_{J} w_{0} \cdot v_{\kappa}=v_{\kappa}$ up to nonzero scalar multiplication, then $b_{1} w_{0} w_{J} w_{0} \cdot v_{\kappa}=v_{\kappa}$ up to nonzero scalar multiplication by a choice of $b_{1}$. Thus $\left\langle u \cdot v_{w_{0} \kappa}, v_{\kappa}\right\rangle \neq 0$. Conversely if $w_{0} w_{J} w_{0} \cdot v_{\kappa} \neq v_{\kappa}$ up to nonzero scalar multiplication, then the action of $B^{-}$on $w_{0} w_{J} w_{0} \cdot v_{\kappa}$ cannot have the highest weight vector $v_{\kappa}$, and hence $\left\langle u \cdot v_{w_{0} \cdot \kappa}, v_{\kappa}\right\rangle=0$. Note that different choices of $w_{0}$ and $w_{J}$ do not affect the result.

Lemma 4.4. Fix $u \in\left(U^{+}\right)^{e}$, and let $r_{1}, \ldots, r_{n}$ be positive integers. Define $J_{u}:=$ $\left\{i \in I \mid \triangle_{r_{i} \kappa_{i}}(u)=0\right\}$. Then $u$ is an element of $B^{-} w^{J} B^{-}$precisely when $J=w_{0} J_{u}$, where the action of $w_{0}$ on $I$ is defined via the Weyl involution on the set of simple roots.

Proof. Suppose $u \in B^{-} w^{J} B^{-}$. We will show that $J=w_{0} J_{u}$. By Lemma 4.3. $i \notin J_{u}$, i.e., $\triangle_{r_{i} \kappa_{i}}(u) \neq 0$ precisely if $w_{0} w_{J} w_{0} \cdot\left(r_{i} \kappa_{i}\right)=r_{i} \kappa_{i}$, which is equivalent to saying

$$
w_{0} w_{J} w_{0} \in\left\{w \in W \mid w \cdot\left(r_{i} \kappa_{i}\right)=r_{i} \kappa_{i}\right\}=\left\{w \in W \mid w \cdot \kappa_{i}=\kappa_{i}\right\}=\left\langle s_{j} \mid j \neq i\right\rangle .
$$

This is possible only when $i \notin w_{0} J$. Therefore $J_{u}=w_{0} J$, i.e., $J=w_{0} J_{u}$. For the converse, suppose $J=w_{0} J_{u}$. Since $u$ is an element of $\left(U^{+}\right)^{e}$, by Lemma 4.2, $u \in B^{-} w^{J^{\prime}} B^{-}$for some $J^{\prime} \subset I$. Applying the same argument as above, we get $J^{\prime}=w_{0} J_{u}$. Since $J=w_{0} J_{u}$, we get $J^{\prime}=J$.

Note that from the proof the set $J_{u}$ does not depend on the choices of $r_{1}, \ldots, r_{n}$. 
Corollary 4.5. As a set, $\overline{B^{-} w^{J} B^{-}} \cap\left(U^{+}\right)^{e}=\left\{u \in\left(U^{+}\right)^{e} \mid \triangle_{r_{i} \kappa_{i}}(u)=0\right.$ for all $\left.i \in w_{0} J\right\}$.

Proof. $u \in \overline{B^{-} w^{J} B^{-}} \cap\left(U^{+}\right)^{e}$ precisely if $u \in\left(B^{-} w^{J^{\prime}} B^{-}\right) \cap\left(U^{+}\right)^{e}$ for some $J^{\prime}$ with $J \subseteq J^{\prime} \subseteq I$ if and only if $w_{0} J^{\prime}=J_{u}$ for some $J^{\prime}$ with $J \subseteq J^{\prime} \subseteq I$ by Lemma 4.4. By the definition of $J_{u}$, this is equivalent to saying that $\triangle_{r_{i} \kappa_{i}}(u)=0$ for all $i \in w_{0} J$.

In the rest of the section, using Corollary 4.5. we compare two presentations of the quantum cohomology rings of $O G^{o}(n), L G(n)$ and $O G^{e}(n)$. Here we remark that the equations cutting the varieties $\mathcal{Y}_{P}$ and $\mathcal{V}_{P}$ may not define reduced schemes, but in our cases we will consider the underlying varieties with the reduced structure, following Rietsch's idea ([19]).

Let

$$
M(m):=\left(\begin{array}{cccccc}
1 & X_{1} & X_{2} & \cdots & X_{m-2} & X_{m-1} \\
& 1 & X_{1} & & & X_{m-2} \\
& & \ddots & \ddots & & \vdots \\
& & & \ddots & X_{1} & X_{2} \\
& & & & 1 & X_{1} \\
& & & & & 1
\end{array}\right)
$$

and let

$$
X_{i, i}:=X_{i}^{2}+2 \sum_{k=1}^{i}(-1)^{k} X_{i+k} X_{i-k}
$$

where $X_{0}=1$

4.2. Case of $O G^{o}(n)$. We fix the nilpotent element $e$ of $\mathfrak{s p}_{2 n}(\mathbb{C})$ :

$$
e=\sum_{i=1}^{n} e_{i}=\sum_{i=1}^{2 n-1} E_{i, i+1} .
$$

The elements $u$ of $\left(U^{+}\right)^{e}$ in $S p_{2 n}(\mathbb{C})$ are exactly matrices of the form $M(2 n)$ in (4.8) together with the relations, $X_{i, i}=0, i=1, \ldots, n-1$. Therefore we can identify the coordinate ring of $\left(U^{+}\right)^{e}$ with a quotient of $\mathbb{C}\left[X_{1}, \ldots, X_{2 n-1}\right]$ modulo the relations $X_{i, i}=0, i=1, \ldots, n-1$.

Definition. We fix the subset $J=\{1, \ldots, n-1\} \subset\{1,2, \ldots, n\}=I$. Define $\mathcal{V}_{n}^{C}$ to be a closed subvariety

$$
\mathcal{V}_{n}^{C}:=\left(U^{+}\right)^{e} \cap \overline{B^{-} w^{J} B^{-}} \subset\left(U^{+}\right)^{e} \subset S p_{2 n}(\mathbb{C}) .
$$

Theorem 4.6 (Peterson). Let $\mathfrak{I}_{o}$ be the ideal of the ring $\mathbb{C}\left[\tau_{1}, \ldots, \tau_{n}\right]$ generated by $\tau_{i, i}$ for $i=1, \ldots, n-1$, and let $\mathfrak{R}_{o}:=\mathbb{C}\left[\tau_{1}, \ldots, \tau_{n}\right] / \mathfrak{I}_{o}$. Then the map $\phi_{C}: \mathfrak{R}_{o} \rightarrow$ $\mathcal{O}\left(\mathcal{V}_{n}^{C}\right)$ defined by $\tau_{i} \mapsto \frac{1}{2} X_{i}$ for $i=1, \ldots, n$ is an isomorphism.

Remark. Note that the ring $\mathfrak{R}_{o}$ is isomorphic to the quantum cohomology ring $q H_{\mathbb{C}}^{*}\left(O G^{e}(n)\right)$ given in $\$ 3.2$, which is in turn isomorphic to $q H_{\mathbb{C}}^{*}\left(O G^{o}(n)\right)$. Therefore 
Theorem 4.6 implies that $q H_{\mathbb{C}}^{*}\left(O G^{o}(n)\right)$ is isomorphic to the reduced coordinate ring $\mathcal{O}\left(\mathcal{V}_{n}^{C}\right)$. The next lemma is a part of the proof of this theorem, and, in 6.2 , we will complete the proof of the theorem by showing that $\mathfrak{R}_{o}$ is reduced.

Lemma 4.7. The map from $\mathbb{C}\left[\tau_{1}, \ldots, \tau_{n}\right]$ to $\mathcal{O}\left(\mathcal{V}_{n}^{C}\right)$ defined by $\tau_{i} \mapsto \frac{1}{2} X_{i}$ for $i=$ $1, \ldots, n$ is a surjective ring homomorphism with kernel the radical of the ideal generated by $\tau_{i, i}, i=1, \ldots, n-1$, with $\tau_{i, i}$ given in $\$ 3.2$.

Proof. Since $J=w_{0} \cdot J=\{1, \ldots, n-1\}$, by Corollary 4.5 ,

$$
\mathcal{V}_{n}^{C}=\left\{u \in\left(U^{+}\right)^{e} \mid \triangle_{\kappa_{r}}(u)=0, r=1,2, \ldots, n-1\right\}
$$

For each $r=1, \ldots, n-1$,

$$
\kappa_{r}=l_{1}+\cdots+l_{r} \quad \text { and } \quad w_{0} \cdot \kappa_{r}=-l_{1}-\cdots-l_{r}
$$

and their weight vectors are

$$
\xi_{1} \wedge \cdots \wedge \xi_{r} \quad \text { and } \quad \xi_{2 n+1-r} \wedge \cdots \wedge \xi_{2 n}
$$

respectively. Therefore $\triangle_{\kappa_{r}}(u)$ is the determinant of the $(r \times r)$ submatrix on the right upper corner of the matrix $M(2 n)$. The vanishing of the determinant $\triangle_{\kappa_{r}}(u)$, $r=1, \ldots, n-1$, inductively implies that the coordinates $X_{2 n-1}, \ldots, X_{n+1}$ vanish. This proves the lemma.

We note that $\mathcal{V}_{n}^{C}$ is a 1-dimensional closed subvariety of $\left(U^{+}\right)^{e}$ because the dimension of $\left(U^{+}\right)^{e}$ is $n([7])$, and it is cut out by $(n-1)$ coordinate functions $X_{n+1}, X_{n+2}, \ldots, X_{2 n-1}$.

4.3. Case of $L G(n)$. We recall that the special result of Peterson holds for minuscule parabolic subgroups, and $P_{n} \subset S p_{2 n}(\mathbb{C})$ is not minuscule (but cominuscule). But we can still find an analogue of Peterson's result for this case. For simplicity, we take $e^{\prime}$ of $\mathfrak{s o}_{2 n+1}(\mathbb{C})$, which is a normalization of the principal nilpotent element $e$ given in $\$ 2.3$.

$$
e^{\prime}:=\sum_{i=1}^{n}\left(E_{i, i+1}+E_{i^{*}-1, i^{*}}\right)=\sum_{i=1}^{2 n} E_{i, i+1},
$$

where $i^{*}=2 n+2-i$. We note that all the lemmas in 4 .1 hold for $e^{\prime}$. The elements $u$ of $\left(U^{+}\right)^{e^{\prime}}$ in $S O_{2 n+1}(\mathbb{C})$ are precisely matrices of the form $M(2 n+1)$ in (4.8) together with $X_{i, i}=0, i=1, \ldots, n$.

Therefore the coordinate ring $\left(U^{+}\right)^{e^{\prime}}$ can be identified with a quotient of the polynomial ring $\mathbb{C}\left[X_{1}, \ldots, X_{2 n}\right]$ modulo the relations $X_{i, i}=0, i=1, \ldots, n$.

Definition. We fix the subset $J=\{1, \ldots, n-1\} \subset\{1,2, \ldots, n\}=I$. Define $\mathcal{V}_{n}^{B}$ to be a closed subvariety

$$
\mathcal{V}_{n}^{B}:=\left(U^{+}\right)^{e^{\prime}} \cap \overline{B^{-} w^{J} B^{-}} \subset\left(U^{+}\right)^{e^{\prime}} \subset S O_{2 n+1}(\mathbb{C})
$$


Theorem 4.8. Let $\mathfrak{I}_{L}$ be the ideal of the polynomial ring $\mathbb{C}\left[\widetilde{Q}_{1}, \ldots, \widetilde{Q}_{n+1}\right]$ generated by $\widetilde{Q}_{i, i}$ for $i=1, \ldots, n$, and let $\Re_{L}:=\mathbb{C}\left[\widetilde{Q}_{1}, \ldots, \widetilde{Q}_{n+1}\right] / \mathfrak{I}_{L}$. Then the map $\phi_{B}: \mathfrak{R}_{L} \rightarrow$ $\mathcal{O}\left(\mathcal{V}_{n}^{B}\right)$ defined by $\widetilde{Q}_{i} \mapsto X_{i}$ for $i=1, \ldots, n+1$ is an isomorphism. Here the $\widetilde{Q}_{i}$ are understood as $\widetilde{Q}_{i}\left(X_{n+1}\right)$.

Remark. We note that the map from $q H_{\mathbb{C}}^{*}(L G(n))$ to $\Re_{L}$ defined by

$$
\begin{aligned}
& \sigma_{i} \mapsto \quad \widetilde{Q}_{i} \quad \text { for } i=1, \ldots, n, \\
& q \mapsto 2 \widetilde{Q}_{n+1}
\end{aligned}
$$

is an isomorphism (9]), and so Theorem 4.8 implies that the quantum cohomology $\operatorname{ring} q H_{\mathbb{C}}^{*}(L G(n))$ is isomorphic to the reduced coordinate $\operatorname{ring} \mathcal{O}\left(\mathcal{V}_{n}^{B}\right)$. The following lemma and 6.2 give a full proof of this theorem.

Lemma 4.9. The map from $\mathbb{C}\left[\widetilde{Q}_{1}, \ldots, \widetilde{Q}_{n+1}\right]$ to $\mathcal{O}\left(\mathcal{V}_{n}^{B}\right)$ defined by $\widetilde{Q}_{i} \mapsto X_{i}$ for $i=1, \ldots, n+1$ is a surjective ring homomorphism with kernel the radical of the ideal generated by $\widetilde{Q}_{i, i}, i=1, \ldots, n$.

Proof. In this case, $J=w_{0} \cdot J=\{1, \ldots, n-1\}$,

$$
\triangle_{\kappa_{r}}(u)=\left\langle u \cdot \xi_{2 n+2-r} \wedge \cdots \wedge \xi_{2 n+1}, \xi_{1} \wedge \cdots \wedge \xi_{r}\right\rangle,
$$

and

$$
\mathcal{V}_{n}^{B}=\left\{u \in\left(U^{+}\right)^{e^{\prime}} \mid \triangle_{\kappa_{r}}(u)=0, r=1, \ldots, n-1\right\} .
$$

Therefore, as in the case of $O G^{o}(n), X_{2 n}, \ldots, X_{n+2}$ inductively vanish. So the lemma follows.

4.4. Case of $O G^{e}(n)$. In this case we take the principal nilpotent element of $\mathfrak{s o}_{2 n+2}(\mathbb{C})$ :

$$
e=\sum_{i=1}^{n+1} e_{i}=\sum_{i=1}^{n}\left(E_{i, i+1}-E_{2 n+2-i, 2 n+3-i}\right)+\left(E_{n, n+2}-E_{n+1, n+3}\right) .
$$

The elements $v$ of $\left(U^{+}\right)^{e}$ in $S O_{2 n+2}(\mathbb{C})$ are exactly matrices of the block form

$$
v=\left(\begin{array}{cc}
A & B \\
O & C
\end{array}\right)
$$

satisfying the following relations (4.11) and (4.12):

$$
X_{r, r}^{\prime}:=X_{r}^{\prime 2}-2 \sum_{i=1}^{r}(-1)^{i+1} X_{r+i}^{\prime} X_{r-i}^{\prime}=0 \text { for } r=1, \ldots, n-1,
$$

where $X_{k}^{\prime}=X_{k}$ if $k \leq n-1$, and $X_{k}^{\prime}=Y_{k}$ otherwise,

$$
X_{n}^{2}-2 \sum_{i=0}^{n} Y_{n+i} X_{n-i}=0 .
$$


The submatrices of $v$ are given by

$$
\begin{aligned}
& A=\left(\begin{array}{cccccc}
1 & X_{1} & X_{2} & \cdots & X_{n-1} & X_{n} \\
0 & 1 & X_{1} & X_{2} & \cdots & X_{n-1} \\
0 & 0 & 1 & X_{1} & \ddots & \vdots \\
\vdots & \vdots & 0 & \ddots & \ddots & X_{2} \\
0 & 0 & 0 & \ddots & 1 & X_{1} \\
0 & 0 & 0 & \cdots & 0 & 1
\end{array}\right), \\
& B=\left(\begin{array}{c|ccccc}
2 Y_{n}-X_{n} & -2 Y_{n+1} & 2 Y_{n+2} & \cdots & (-1)^{n-1} 2 Y_{2 n-1} & (-1)^{n} 2 Y_{2 n} \\
X_{n-1} & -2 Y_{n} & 2 Y_{n+1} & \cdots & (-1)^{n-1} 2 Y_{2 n-2} & (-1)^{n} 2 Y_{2 n-1} \\
X_{n-2} & -2 X_{n-1} & 2 Y_{n} & \cdots & (-1)^{n-1} 2 Y_{2 n-3} & (-1)^{n} 2 Y_{2 n-2} \\
X_{n-3} & -2 X_{n-2} & 2 X_{n-1} & \cdots & (-1)^{n-1} 2 Y_{2 n-4} & (-1)^{n} 2 Y_{2 n-3} \\
\vdots & \vdots & & \vdots & \vdots & \vdots \\
X_{3} & -2 X_{4} & 2 X_{5} & \cdots & (-1)^{n-1} 2 Y_{n+2} & (-1)^{n} 2 Y_{n+3} \\
X_{2} & -2 X_{3} & 2 X_{4} & \cdots & (-1)^{n-1} 2 Y_{n+1} & (-1)^{n} 2 Y_{n+2} \\
X_{1} & -2 X_{2} & 2 X_{3} & \cdots & (-1)^{n-1} 2 Y_{n} & (-1)^{n} 2 Y_{n+1} \\
\hline 0 & -X_{1} & X_{2} & \cdots & (-1)^{n-1} X_{n-1} & W_{n}
\end{array}\right), \\
& C=\left(\begin{array}{cccccc}
1 & -X_{1} & X_{2} & \cdots & (-1)^{n-1} X_{n-1} & Z_{n} \\
0 & 1 & -X_{1} & X_{2} & \ddots & (-1)^{n-1} X_{n-1} \\
0 & 0 & 1 & -X_{1} & \ddots & \vdots \\
\vdots & 0 & 0 & \ddots & \ddots & X_{2} \\
0 & \vdots & \ddots & \ddots & 1 & -X_{1} \\
0 & 0 & \cdots & 0 & 0 & 1
\end{array}\right),
\end{aligned}
$$

and the submatrix $O$ is the $(n+1) \times(n+1)$ zero matrix. Here

$$
W_{n}= \begin{cases}X_{n}-2 Y_{n} & \text { if } n \text { is odd, } \\ X_{n} & \text { if } n \text { is even }\end{cases}
$$

and

$$
Z_{n}=\left\{\begin{array}{cc}
-X_{n} & \text { if } n \text { is odd } \\
2 Y_{n}-X_{n} & \text { if } n \text { is even. }
\end{array}\right.
$$

The coordinate ring of $\left(U^{+}\right)^{e}$ can be identified with a quotient of the ring $\mathbb{C}\left[X_{1}, \ldots, X_{n}, Y_{n}, \ldots, Y_{2 n}\right]$ modulo the relations (4.11) and (4.12).

Definition. For the subset $J=\{1, \ldots, n\} \subset\{1, \ldots, n+1\}=I$, we define $\mathcal{V}_{n+1}^{D}$ to be a closed subvariety $\mathcal{V}_{n+1}^{D}:=\left(U^{+}\right)^{e} \cap \overline{B^{-} w^{J} B^{-}} \subset\left(U^{+}\right)^{e} \subset S O_{2 n+2}(\mathbb{C})$.

Theorem 4.10 (Peterson). There is an isomorphism $\phi_{D}: \mathfrak{R}_{o} \rightarrow \mathcal{O}\left(\mathcal{V}_{n+1}^{D}\right)$ that takes $\tau_{i}$ to $X_{i}^{\prime}, i=1, \ldots, n$.

Note that since the ring $\mathfrak{R}_{o}$ is isomorphic to the quantum cohomology ring $q H_{\mathbb{C}}^{*}\left(O G^{e}(n)\right)$, this theorem implies that $q H_{\mathbb{C}}^{*}\left(O G^{e}(n)\right)$ is isomorphic to the reduced coordinate ring $\mathcal{O}\left(\mathcal{V}_{n+1}^{D}\right)$. The following lemma is a part of the proof of the theorem. 
To complete the proof of the theorem, it remains to show that $\mathfrak{R}_{o}$ is reduced, which will be done in 6.2 .

Lemma 4.11. The map from $\mathbb{C}\left[\tau_{1}, \ldots, \tau_{n}\right]$ to $\mathcal{O}\left(\mathcal{V}_{n+1}^{D}\right)$ defined by $\tau_{i} \mapsto X_{i}^{\prime}$ for $i=1, \ldots, n$ is a surjective ring homomorphism with kernel the radical of the ideal generated by $\tau_{i, i}, i=1, \ldots, n-1$.

Proof. First recall from $\$ 2.4$ that the fundamental weights of $S_{2 n+2}(\mathbb{C})$ consist of $(n+2)$-weights, $\omega_{r}$ for $r=1, \ldots, n, \omega_{n+1}^{-}=2 \kappa_{n}$ and $\omega_{n+1}^{+}=2 \kappa_{n+1}$.

For the sake of simplicity, let

$$
\omega_{n+1}:= \begin{cases}\omega_{n+1}^{-} & \text {if } n \text { is odd } \\ \omega_{n+1}^{+} & \text {if } n \text { is even. }\end{cases}
$$

By (2.3), for $J=\{1, \ldots, n\}$, we have

$$
w_{0} J=\left\{\begin{array}{cc}
\{1, \ldots, n\} & \text { if } n \text { is odd } \\
\{1, \ldots, n-1, n+1\} & \text { if } n \text { is even }
\end{array}\right.
$$

Therefore Corollary 4.5 implies that, for any $n$, odd or even, the variety $\mathcal{V}_{n+1}^{D}$ is defined by the equations $\triangle_{\omega_{k}}(v)$ for $k=1, \ldots, n-1, n+1$, and the coordinate ring $\mathcal{O}\left(\mathcal{V}_{n+1}^{D}\right)$ is isomorphic to the ring

$$
\mathbb{C}\left[X_{1}, . ., X_{n}, Y_{n}, \ldots, Y_{2 n}\right] / \operatorname{Rad}\left(\mathfrak{I}_{0}\right),
$$

where $\operatorname{Rad}\left(\mathfrak{I}_{0}\right)$ is the radical of the ideal $\mathfrak{I}_{0}$, and $\mathfrak{I}_{0}$ is generated by the polynomials in (4.11) and (4.12), and polynomials $\triangle_{\omega_{k}}(v)$ for $k \in\{1, \ldots, n-1, n+1\}$. From the relation (2.2) in 2.4 and the fact that $\triangle_{\omega_{k}}(v)$ lie in $\operatorname{Rad}\left(\Im_{0}\right)$ for $k \in\{1, \ldots, n-$ $1, n+1\}$, the polynomial $\triangle_{\omega_{n}}^{2}(v)$ lies in $\operatorname{Rad}\left(\mathfrak{I}_{0}\right)$, and so does $\triangle_{\omega_{n}}(v)$. For each $k=1, \ldots, n$, the matrix coefficient $\triangle_{\omega_{k}}(v)$ is given by the determinant of the $(k \times k)$ submatrix of $B$ on the right upper corner. Note that if $n$ is odd, then the weights

$$
\omega_{n+1}=l_{1}+\cdots+l_{n}-l_{n+1} \quad \text { and } \quad w_{0} \omega_{n+1}=-l_{1}-\cdots-l_{n}+l_{n+1}
$$

have weight vectors

$$
\xi_{1} \wedge \cdots \xi_{n} \wedge \xi_{n+2} \quad \text { and } \quad \xi_{n+1} \wedge \xi_{n+3} \wedge \cdots \wedge \xi_{2 n+2},
$$

respectively, and if $n$ is even, the weights

$$
\omega_{n+1}=l_{1}+\cdots+l_{n}+l_{n+1} \quad \text { and } \quad w_{0} \omega_{n+1}=-l_{1}-\cdots-l_{n}+l_{n+1}
$$

have weight vectors

$$
\xi_{1} \wedge \cdots \wedge \xi_{n} \wedge \xi_{n+1} \quad \text { and } \quad \xi_{n+1} \wedge \xi_{n+3} \wedge \cdots \wedge \xi_{2 n+2},
$$

respectively. Therefore if $n$ is odd, $\triangle_{\omega_{n+1}}(v)$ is the determinant of a submatrix $M_{n}$ defined below of $v$ with $(1, \ldots, n, n+2)$-th rows and $(n+1, n+3, \ldots, 2 n+2)$-th columns, and if $n$ is even, $\triangle_{\omega_{n+1}}(v)$ is the determinant of the submatrix $M_{n}$ of $v$ with $(1, \ldots, n, n+1)$-th rows and $(n+1, n+3, \ldots, 2 n+2)$-th columns. Here the submatrix $M_{n}$ is given as follows. 


$$
M_{n}=\left(\begin{array}{c|ccccc}
X_{n} & -2 Y_{n+1} & 2 Y_{n+2} & \cdots & (-1)^{n-1} 2 Y_{2 n-1} & (-1)^{n} 2 Y_{2 n} \\
X_{n-1} & -2 Y_{n} & 2 Y_{n+1} & \cdots & (-1)^{n-1} 2 Y_{2 n-2} & (-1)^{n} 2 Y_{2 n-1} \\
X_{n-2} & -2 X_{n-1} & 2 Y_{n} & \cdots & (-1)^{n-1} 2 Y_{2 n-3} & (-1)^{n} 2 Y_{2 n-2} \\
X_{n-3} & -2 X_{n-2} & 2 X_{n-1} & \cdots & (-1)^{n-1} 2 Y_{2 n-4} & (-1)^{n} 2 Y_{2 n-3} \\
\vdots & \vdots & \vdots & & \vdots & \vdots \\
X_{3} & -2 X_{4} & 2 X_{5} & \cdots & (-1)^{n-1} 2 Y_{n+2} & (-1)^{n} 2 Y_{n+3} \\
X_{2} & -2 X_{3} & 2 X_{4} & \cdots & (-1)^{n-1} 2 Y_{n+1} & (-1)^{n} 2 Y_{n+2} \\
X_{1} & -2 X_{2} & 2 X_{3} & \cdots & (-1)^{n-1} 2 Y_{n} & (-1)^{n} 2 Y_{n+1} \\
\hline a & -X_{1} & X_{2} & \cdots & (-1)^{n-1} X_{n-1} & P_{n}
\end{array}\right),
$$

where

and

$$
P_{n}=\left\{\begin{array}{cc}
Z_{n} & \text { if } n \text { is odd } \\
W_{n} & \text { if } n \text { is even, }
\end{array}\right.
$$

$$
a= \begin{cases}0 & \text { if } n \text { is odd, } \\ 1 & \text { if } n \text { is even. }\end{cases}
$$

The containment of $\triangle_{\omega_{k}}(v)$ in $\operatorname{Rad}\left(\mathfrak{I}_{0}\right)$ for $k=1, \ldots, n$ implies inductively that $Y_{2 n}, \ldots, Y_{n+1}$ lie in the ideal $\operatorname{Rad}\left(\mathfrak{I}_{0}\right)$. Therefore the ring in (4.13) is isomorphic to

$$
\mathbb{C}\left[X_{1}, \ldots, X_{n}, Y_{n}\right] / \operatorname{Rad}\left(\Im_{1}\right) .
$$

Here the ideal $\Im_{1}$ is generated by the following polynomials (4.15), (4.16) and (4.17):

$$
\begin{aligned}
& X_{r}^{\prime 2}-2 \sum_{i=1}^{r}(-1)^{i+1} X_{r+i}^{\prime} X_{r-i}^{\prime} \text { for } r=1, \ldots, n-1, \\
& X_{n}^{2}-2 X_{n} Y_{n}, \\
& \left.\triangle_{\omega_{n+1}}(v)\right|_{Y_{2 n}=\cdots=Y_{n+1}=0 .} .
\end{aligned}
$$

From the matrix $M_{n}$, we easily see that $\left.\triangle_{\omega_{n+1}}(v)\right|_{Y_{2 n}=\cdots=Y_{n+1}=0}=$ $(-1)^{n} 2^{n-1} X_{n}^{2} Y_{n}^{n-1}$ for $n$, both odd and even. From (4.16) and (4.17), it follows that in the ring $\mathbb{C}\left[X_{1}, \ldots, X_{n}, Y_{n}\right] / \operatorname{Rad}\left(\Im_{1}\right)$, we have the relations

$$
X_{n}^{2}=2 X_{n} Y_{n} \text { and } X_{n}^{2} Y_{n}^{n-1}=0 .
$$

This implies

$$
X_{n}^{2 n+1}=2^{n} X_{n}^{n+1} Y_{n}^{n}=2^{n}\left(X_{n}^{n-1} Y_{n}\right)\left(X_{n}^{2} Y_{n}^{n-1}\right)=0 .
$$

Since the ring $\mathbb{C}\left[X_{1}, \ldots, X_{n}, Y_{n}\right] / \operatorname{Rad}\left(\mathfrak{I}_{1}\right)$ is reduced, we have $X_{n}=0$ in the ring $\mathbb{C}\left[X_{1}, \ldots, X_{n}, Y_{n}\right] / \operatorname{Rad}\left(\Im_{1}\right)$. Therefore the coordinate ring $\mathcal{O}\left(\mathcal{V}_{n+1}^{D}\right)$ is isomorphic to the following reduced ring:

$$
\mathbb{C}\left[X_{1}^{\prime}, \ldots, X_{n}^{\prime}\right] / \operatorname{Rad}\left(\mathfrak{I}_{2}\right),
$$

where the ideal $\mathfrak{I}_{2}$ is generated by the polynomials

$$
X_{r}^{\prime 2}-2 \sum_{i=1}^{r}(-1)^{i+1} X_{r+i}^{\prime} X_{r-i}^{\prime} \text { for } r=1, \ldots, n-1 .
$$

This proves the lemma.

Remark. From Lemma 4.7, we have a well-defined map (a priori not an isomorphism) $\phi_{C}: \mathfrak{R}_{o} \rightarrow \mathcal{O}\left(\mathcal{V}_{n}^{C}\right)$. So each element $F$ of $\mathfrak{R}_{o}$ induces a function $\phi_{C}(F)$ on $\mathcal{V}_{n}^{C}$. We will write $\dot{F}$ for $\phi_{C}(F)$. Similarly, $\phi_{B}(G):=\dot{G}$ and $\phi_{D}(H)=\dot{H}$ can be considered as functions on $\mathcal{V}_{n}^{B}$ and $\mathcal{V}_{n+1}^{D}$ for any $G \in \mathfrak{R}_{L}$ and $H \in \mathfrak{R}_{o}$. 


\section{Elements of VARIETIES $\mathcal{V}_{n}^{C}, \mathcal{V}_{n}^{B}$ AND $\mathcal{V}_{n+1}^{D}$}

In this section, we describe elements of $\mathcal{V}_{n}^{C}, \mathcal{V}_{n}^{B}$ and $\mathcal{V}_{n+1}^{D}$. To do this, we need the following definitions and notation.

5.1. Definitions and notation. Let us call $I=\left(i_{1}, \ldots, i_{n}\right) \in \mathcal{T}_{n}$ exclusive if $\zeta^{i_{k}} \neq-\zeta^{i_{l}}$ for all $k, l=1, \ldots, n$. So if $I$ is exclusive, then the set $\left\{\zeta^{i_{1}}, \ldots, \zeta^{i_{n}}\right\}$ cannot have both of two antipodal points, but only one. We also call $I=\left(i_{1}, \ldots, i_{n}\right) \in \mathcal{T}_{n}$ self-symmetric if the set $\left\{\zeta^{i_{1}}, \ldots, \zeta^{i_{n}}\right\}$ contains both $\zeta^{i_{k}}$ and the complex conjugate $\overline{\zeta^{i_{k}}}$. Recall that $\mathcal{I}_{n}$ denotes the set of all exclusive $n$-tuples $I=\left(i_{1}, \ldots, i_{n}\right)$. Denote by $\mathcal{I}_{n}^{s}$ the set of exclusive and self-symmetric $n$-tuples $I=\left(i_{1}, \ldots, i_{n}\right)$ in $\mathcal{T}_{n}$. We can easily check that $\left|\mathcal{I}_{n}\right|=2^{n}=|\mathcal{D}(n)|$ and $\left|\mathcal{I}_{n}^{s}\right|=2^{\left\lfloor\frac{n}{2}\right\rfloor}$.

For $a_{1}, \ldots, a_{n} \in \mathbb{C}$, define a matrix $\tilde{u}_{m}\left(a_{1}, \ldots, a_{n}\right) \in S L_{m}(\mathbb{C})$ as

$$
\tilde{u}_{m}\left(a_{1}, \ldots, a_{n}\right):=\left(\begin{array}{cccccccc}
1 & a_{1} & a_{2} & \cdots & a_{n} & 0 & \cdots & 0 \\
& 1 & a_{1} & \ddots & & \ddots & & \vdots \\
& & 1 & \ddots & & & \ddots & 0 \\
& & & \ddots & & & & a_{n} \\
& & & & \ddots & \ddots & \ddots & \vdots \\
& & & & & 1 & a_{1} & a_{2} \\
& & & & & & 1 & a_{1} \\
& & & & & & & 1
\end{array}\right) .
$$

For $x_{1}, \ldots, x_{n} \in \mathbb{C}$, let $u_{m}\left(x_{1}, \ldots, x_{n}\right):=\tilde{u}_{m}\left(E_{1}\left(x_{1}, \ldots, x_{n}\right), \ldots, E_{n}\left(x_{1}, \ldots, x_{n}\right)\right)$. Similarly, let $\tilde{v}_{2 n+2}\left(a_{1}, \ldots, a_{n}\right)$ be the matrix $v$ in (4.10) with $X_{i}^{\prime}$ replaced by $a_{i}$ for $i=1, \ldots, n$, and with $X_{n}$ and other $X_{i}^{\prime}$ replaced by 0 . Let $v_{2 n+2}\left(x_{1}, \ldots, x_{n}\right):=$ $\tilde{v}_{2 n+2}\left(E_{1}\left(x_{1}, \ldots, x_{n}\right), \ldots E_{n}\left(x_{1}, \ldots, x_{n}\right)\right)$.

We suppress the subscripts in $u_{m}$ and $v_{2 n+2}$ if any confusion does not arise.

5.2. Elements of varieties $\mathcal{V}_{n}^{C}, \mathcal{V}_{n}^{B}$ and $\mathcal{V}_{n+1}^{D}$. We now characterize the elements of $\mathcal{V}_{n}^{C}, \mathcal{V}_{n}^{B}$ and $\mathcal{V}_{n+1}^{D}$ in terms of $x_{i}$.

Lemma 5.1. For $I=\left(i_{1}, \ldots, i_{n}\right) \in \mathcal{T}_{n}, I$ is exclusive precisely when $E_{i}\left(\zeta^{2 I}\right)=0$ for all $i=1, \ldots, n-1$.

Proof. Obviously, $I_{0}:=\left(-\frac{n-1}{2},-\frac{n-1}{2}+1, \ldots, \frac{n-1}{2}\right)$ is exclusive, $E_{i}\left(\zeta^{2 I_{0}}\right)=0$ for $i=1, \ldots, n-1$ and $E_{n}\left(\zeta^{2 I_{0}}\right)=1$. If $I$ is exclusive, then $2 I=2 I_{0}$ up to order, and so $E_{i}\left(\zeta^{2 I}\right)=0$ for $i=1, \ldots, n-1$. For the converse, suppose $E_{i}\left(\zeta^{2 I}\right)=0$ for $i=1, \ldots, n-1$. If $I$ is not exclusive, then there are $k$ and $l$ such that $\zeta^{2 i_{k}}=\zeta^{2 i_{l}}$. Consider the following polynomial in $z$, of which the zeros are $\eta_{m}:=\zeta^{2 i_{m}}$ for $m=1, \ldots, n$ :

$$
g(z)=\prod_{i=1}^{n}\left(z-\eta_{i}\right)
$$

Since $E_{i}\left(\eta_{1}, \ldots, \eta_{n}\right)=0$ for $i=1, \ldots, n-1$, we have

$$
\prod_{i=1}^{n}\left(z-\eta_{i}\right)=z^{n}+(-1)^{n} E_{n}\left(\eta_{1}, \ldots, \eta_{n}\right) \text {. }
$$


If we take derivatives and evaluation at $z=\eta_{k}$ of both sides of (5.21), the left hand side is zero, and the right hand side is $n \eta_{k}^{n-1}$, which is nonzero, which yields a contradiction.

Lemma 5.2. The matrix $u_{2 n}\left(x_{1}, \ldots, x_{n}\right)$ is an element of $\mathcal{V}_{n}^{C}$ if and only if $\left(x_{1}, \ldots, x_{n}\right)$ can be written (up to order) as $\left(x_{1}, \ldots, x_{n}\right)=t \zeta^{I}$ for some $I \in \mathcal{I}_{n}$ and $t \in \mathbb{C}$. All elements of $\mathcal{V}_{n}^{C}$ are of the form $u_{2 n}\left(t \zeta^{I}\right)$ with $t \in \mathbb{C}$ and $I \in \mathcal{I}_{n}$.

Proof. First note that from Lemma 4.7 all the elements of $\mathcal{V}_{n}^{C}$ are of the form $u_{2 n}\left(x_{1}, \ldots, x_{n}\right)$ for complex numbers $x_{1}, \ldots, x_{n}$, and, by the definitions of $X_{i, i}$ and $u_{2 n}\left(x_{1}, \ldots, x_{n}\right)$, we have

$$
X_{i, i}\left(u_{2 n}\left(x_{1}, \ldots, x_{n}\right)\right)=\widetilde{Q}_{i, i}\left(x_{1}, \ldots, x_{n}\right),
$$

which is equal to $E_{i}\left(x_{1}^{2}, \ldots, x_{n}^{2}\right)$ by the property (1) of $\widetilde{Q}$-polynomials in $\$ 2.5$. Therefore $\mathcal{V}_{n}^{C}$ consists of all $u_{2 n}\left(x_{1}, \ldots, x_{n}\right)$ such that $E_{i}\left(x_{1}^{2}, \ldots, x_{n}^{2}\right)=0, i=1, \ldots, n-1$. To prove the lemma, it suffices to show that $E_{i}\left(x_{1}^{2}, \ldots, x_{n}^{2}\right)=0$ for $i=1, \ldots, n-1$ if and only if $\left(x_{1}, \ldots, x_{n}\right)=t \zeta^{I}$ for some $I \in \mathcal{I}_{n}$ and $t \in \mathbb{C}$. This holds trivially for $\left(x_{1}, \ldots, x_{n}\right)=(0, \ldots, 0)$. So we assume $\left(x_{1}, \ldots x_{n}\right) \neq(0, \ldots, 0)$. First suppose that $E_{i}\left(x_{1}^{2}, \ldots, x_{n}^{2}\right)=0$ for all $i=1, \ldots, n-1$. Consider the following polynomial in $z$ :

$$
f(z)=\prod_{i=1}^{n}\left(z-x_{i}^{2}\right) .
$$

Since $E_{i}\left(x_{1}^{2}, \ldots, x_{n}^{2}\right)=0$ for $i=1, \ldots, n-1$, we have

$$
\prod_{i=1}^{n}\left(z-x_{i}^{2}\right)=z^{n}+(-1)^{n} E_{n}\left(x_{1}^{2}, . ., x_{n}^{2}\right) .
$$

But since $x_{1}^{2}, \ldots, x_{n}^{2}$ are roots of $f(z)$, we have

$$
x_{1}^{2 n}=\cdots=x_{n}^{2 n}=(-1)^{n+1} E_{n}\left(x_{1}^{2}, \ldots, x_{n}^{2}\right) .
$$

If $x_{i}=0$ for some $1 \leq i \leq n$, then trivially all $x_{i}$ are zero (5.23), which contradicts the assumption that $\left(x_{1}, \ldots x_{n}\right) \neq(0, \ldots, 0)$, and hence all $x_{i}$ are nonzero. Furthermore all $x_{i}$ are distinct. Indeed if all $x_{i}$ are not distinct, say, if $x_{1}=x_{2}$, taking derivatives and evaluation at $z=x_{1}^{2}$ of both sides of (5.22), the left hand side is zero and the right hand side is $n x_{1}^{2 n-2}$, which is nonzero, and hence we get a contradiction. Now by the description (5.23) of $x_{1}, \ldots, x_{n}$, we can write $\left(x_{1}, \ldots, x_{n}\right)=t \zeta^{I}$ for some $I \in \mathcal{T}_{n}$ and nonzero $t \in \mathbb{C}$. But since

$$
E_{i}\left(x_{1}^{2}, \ldots, x_{n}^{2}\right)=E_{i}\left(t^{2} \zeta^{2 I}\right)=t^{2 i} E_{i}\left(\zeta^{2 I}\right)=0,
$$

and $t$ is nonzero, we have $E_{i}\left(\zeta^{2 I}\right)=0$, and so, by Lemma 5.1, $I$ is exclusive. The other direction is trivial by Lemma 5.1. This completes the proof.

Lemma 5.3. The matrix $u_{2 n+1}\left(x_{1}, \ldots x_{n+1}\right)$ is an element of $\mathcal{V}_{n}^{B}$ if and only if $\left(x_{1}, \ldots, x_{n+1}\right)$ can be written (up to order) as $\left(x_{1}, \ldots, x_{n+1}\right)=t \zeta^{I}$ for some $I \in \mathcal{I}_{n+1}$ and $t \in \mathbb{C}$. All elements of $\mathcal{V}_{n}^{B}$ are of the form $u_{2 n+1}\left(t \zeta^{I}\right)$ with $t \in \mathbb{C}$ and $I \in \mathcal{I}_{n+1}$.

Proof. The same argument as in the proof of Lemma 5.2 applies, with " $u_{2 n}\left(x_{1}, \ldots, x_{n}\right)$ " and " $X_{i, i}=0$ for $i=1, \ldots, n-1$ " replaced by " $u_{2 n+1}\left(x_{1}, \ldots, x_{n+1}\right)$ " and " $X_{i, i}=0$ for $i=1, \ldots, n$ ", respectively. 
Lemma 5.4. The matrix $v_{2 n+2}\left(x_{1}, \ldots x_{n}\right)$ is an element of $\mathcal{V}_{n+1}^{D}$ if and only if $\left(x_{1}, \ldots, x_{n}\right)$ can be written (up to order) as $\left(x_{1}, \ldots, x_{n}\right)=t \zeta^{I}$ for some $I \in \mathcal{I}_{n}$ and $t \in \mathbb{C}$. All elements of $\mathcal{V}_{n+1}^{D}$ are of the form $v_{2 n+2}\left(t \zeta^{I}\right)$ with $t \in \mathbb{C}$ and $I \in \mathcal{I}_{n}$.

Proof. The argument of the proof of Lemma 5.2 applies, with " $u_{2 n}\left(x_{1}, \ldots, x_{n}\right)$ " and " $X_{i, i}=0$ for $i=1, \ldots, n-1$ " replaced by " $v_{2 n+2}\left(x_{1}, \ldots, x_{n}\right)$ " and " $X_{i, i}^{\prime}=0$ for $i=1, \ldots, n-1$ ", respectively.

The evaluations of the functions $\dot{q}$ defined by the quantum variables will be used to find the Vafa-Intriligator type formulas in Section 7 .

Lemma 5.5. (1) For the quantum variable $q$ for $O G^{o}(n)$ and $u=u_{2 n}\left(t \zeta^{I}\right) \in$ $\mathcal{V}_{n}^{C}$, we have

$$
\dot{q}(u)=\frac{1}{4} t^{2 n} .
$$

(2) For the quantum variable $q$ for $L G(n)$ and $u=u_{2 n+1}\left(t \zeta^{I}\right) \in \mathcal{V}_{n}^{B}$, we have

$$
\dot{q}(u)=2 t^{n+1} E_{n+1}\left(\zeta^{I}\right) \text { and } \dot{q}^{2}(u)=4 t^{2 n+2} .
$$

Proof. (1) follows directly from the fact that $q=\tau_{n}^{2}$ corresponds to $\widetilde{P}_{n}^{2}$ by $\$ 3.2$, and

$$
\widetilde{P}_{n, n}\left(x_{1}, \ldots, x_{n}\right)=\frac{1}{4} E_{n}\left(x_{1}^{2}, \ldots, x_{n}^{2}\right)=(-1)^{n+1} \frac{1}{4} x_{i}^{2 n} .
$$

Since $q$ corresponds to $2 E_{n+1}$, we get (2).

\subsection{The varieties $\mathcal{V}_{n}^{B}, \mathcal{V}_{n}^{C}$ and $\mathcal{V}_{n+1}^{D}$. Let}

$$
U_{2 n}:=\left\{z \in \mathbb{C} \mid z^{2 n}=1\right\}=\left\{\zeta^{i} \mid i=0, \ldots, 2 n-1\right\} .
$$

Consider the natural actions of the groups $U_{2 n}$ and $\mathbb{Z}_{2}$ on the sets $\left\{\zeta^{I} \mid I \in \mathcal{I}_{n}\right\}$ and $\left\{\zeta^{I} \mid I \in \mathcal{I}_{n}^{s}\right\}$, respectively. Let $O_{n}$ and $o_{n}$ be the sets of orbits for the actions $U_{2 n}$ and $\mathbb{Z}_{2}$, respectively. Then we count

$$
\left|O_{n}\right|=\frac{1}{2 n} \sum_{\substack{d \mid n \\ d \text { odd }}} \varphi(d) 2^{\frac{n}{d}} \quad \text { and } \quad\left|o_{n}\right|=2^{\left\lfloor\frac{n}{2}\right\rfloor-1},
$$

where $\varphi(d)$ is the number of positive integers $k \leq d$ such that $k$ is relatively prime to $d$. For $I \in \mathcal{I}_{n}$, let $\mathbb{C}_{I}^{*}:=\left\{u_{2 n}\left(t \zeta^{I}\right) \in \mathcal{V}_{n}^{C} \mid t \in \mathbb{C}^{*}\right\}$, and for $I \in \mathcal{I}_{n}^{s}$, let $\mathbb{R}_{I}^{*}:=\left\{u_{2 n}\left(t \zeta^{I}\right) \in \mathcal{V}_{n}^{C} \mid t \in \mathbb{R}^{*}\right\}$. Then we have

$$
\mathcal{V}_{n}^{C}=\{i d\} \cup \bigcup_{I \in \mathcal{I}_{n}} \mathbb{C}_{I}^{*} \quad \text { and } \quad \mathcal{V}_{n}^{C}(\mathbb{R})=\{i d\} \cup \bigcup_{I \in \mathcal{I}_{n}^{s}} \mathbb{R}_{I}^{*},
$$

where $\mathcal{V}_{n}^{C}(\mathbb{R})$ is the set of real points in $\mathcal{V}_{n}^{C}$, and $i d$ is the identity matrix of $S p_{2 n}(\mathbb{C})$. Note that $\mathbb{C}_{I}^{*}=\mathbb{C}_{J}^{*}$ if and only if $\zeta^{I}$ and $\zeta^{J}$ are in the same orbit. Similarly, $\mathbb{R}_{I}^{*}=\mathbb{R}_{J}^{*}$ if and only if $\zeta^{I}$ and $\zeta^{J}$ are in the same orbit. Therefore there are $\left|O_{n}\right|$-complex curves and $\left|o_{n}\right|$-real curves in $\mathcal{V}_{n}^{C}$, both ramified at the identity matrix $i d \in S p_{2 n}(\mathbb{C})$ $(t=0)$. The situation with $\mathcal{V}_{n+1}^{D}$ is exactly the same. The same arguments also apply to the cases $\mathcal{V}_{n}^{B}$, and so there are $\left|O_{n+1}\right|$-complex curves and $\left|o_{n+1}\right|$-real curves in $\mathcal{V}_{n}^{B}$, both ramified at the identity matrix $i d \in S O_{2 n+1}(\mathbb{C})(t=0)$. 


\section{Evaluations of SYMmEtric FUnCtions AT ROOTS OF UNITY}

In this section, we set up the orthogonality formulas of $\widetilde{Q}$ - and $\widetilde{P}$-polynomials at roots of unity, complete the proof of Theorems 4.6. 4.8 and 4.10, using these orthogonality formulas, and determine on which $n$-tuples of roots of unity all $\widetilde{P}$ polynomials are positive.

6.1. Orthogonality formulas for $\tilde{Q}$ - and $\tilde{P}$-polynomials. For $I \in \mathcal{T}_{n}$, there is a unique $n$-tuple $\left(\hat{i}_{1}, \ldots, \hat{i}_{n}\right) \in \mathcal{T}_{n}$ such that the two sets $\left\{\zeta^{i_{1}}, \ldots \zeta^{i_{n}}\right\}$ and $\left\{\zeta^{\hat{i}_{1}}, \ldots, \zeta^{\hat{i}_{n}}\right\}$ enumerate all roots of $(-1)^{n+1}$. Denote $\hat{I}=\left(\hat{i}_{1}, \ldots, \hat{i}_{n}\right)$.

Lemma 6.1 ([19]). Let $E_{k}$ and $H_{k}$ be the elementary and complete symmetric polynomials, respectively. Then for $I, J \in \mathcal{I}_{n}$, we have the following identities:

(1) $\prod_{k=1}^{n} \prod_{l=1}^{n}\left(1-\zeta^{i_{k}} \zeta^{-\hat{j}_{l}}\right)=\delta_{I, J} \frac{(2 n)^{n}}{\left|\operatorname{Vand}\left(\zeta^{I}\right)\right|^{2}}$,

(2) $E_{k}\left(\zeta^{-I}\right)=H_{k}\left(\zeta^{I^{*}}\right)$,

where $\operatorname{Vand}\left(\zeta^{J}\right):=\prod_{k<l}\left(\zeta^{j_{k}}-\zeta^{j_{l}}\right)$.

Proof. For the proof of these identities, we refer to Proposition 4.3 and Lemma 4.4 in [19].

The next Proposition 6.2 is the analogue for $\widetilde{Q}$ - and $\widetilde{P}$-polynomials of Propositions 4.3 and 6.1 in [19.

Proposition 6.2. Let $I, J \in \mathcal{I}_{n}$ and $z_{1}, \ldots, z_{n}, t \in \mathbb{C}$. Then we have

(1) $\sum_{\lambda \in \mathcal{R}(n)} P_{\lambda}\left(\zeta^{J^{*}}\right) \widetilde{Q}_{\lambda}\left(z_{1}, \ldots, z_{n}\right)=\prod_{k=1}^{n} \prod_{l=1}^{n}\left(1-z_{k} \zeta^{-\hat{j}_{l}}\right)$,

(2) $\sum_{\lambda \in \mathcal{R}(n)} P_{\lambda}\left(\zeta^{J^{*}}\right) \widetilde{Q}_{\lambda}\left(\zeta^{I}\right)=\delta_{I, J} \frac{(2 n)^{n}}{\left|\operatorname{Vand}\left(\zeta^{I}\right)\right|^{2}}$,

(3) $\sum_{\lambda \in \mathcal{R}(n)} \overline{\omega\left(P_{\lambda}\right)\left(\zeta^{J}\right)} \widetilde{Q}_{\lambda}\left(\zeta^{I}\right)=\delta_{I, J} \frac{(2 n)^{n}}{\left|\operatorname{Vand}\left(\zeta^{I}\right)\right|^{2}}$,

(4) $\sum_{\lambda \in \mathcal{D}(n)} \widetilde{P}_{\lambda}\left(\zeta^{I}\right) \widetilde{P}_{\hat{\lambda}}\left(\zeta^{J}\right)=\delta_{I, J} S_{\rho_{n}}\left(\zeta^{I}\right)$,

where $\omega$ is the involution on the ring of symmetric polynomials interchanging the elementary and the complete symmetric polynomials, () stands for complex conjugation, and $\rho_{n}:=(n, n-1, \ldots, 1)$.

Proof. We have the following identity from p. 234 in [14:

$$
\sum_{\lambda \in \mathcal{R}(n)} P_{\lambda}\left(z_{1}, \ldots, z_{n}\right) Q_{\lambda}^{\prime}\left(w_{1}, \ldots, w_{n}\right)=\prod_{k=1}^{n} \prod_{l=1}^{n} \frac{1}{\left(1-z_{k} w_{l}\right)},
$$

where $Q_{\lambda}^{\prime}$ are the Hall-Littlewood functions ([14, Proposition 4.9 in [18]). Note that from the definition of $Q_{\lambda}^{\prime}$, we have $\omega\left(Q_{\lambda}^{\prime}\right)=\widetilde{Q}_{\lambda}$. Therefore if we take the involution $\omega$ with respect to $W=\left(w_{1}, \ldots, w_{n}\right)$ on both sides of (6.24), we have

$$
\sum_{\lambda \in \mathcal{R}(n)} P_{\lambda}\left(z_{1}, \ldots, z_{n}\right) \widetilde{Q}_{\lambda}\left(w_{1}, \ldots, w_{n}\right)=\prod_{k=1}^{n} \prod_{l=1}^{n}\left(1+z_{k} w_{l}\right) .
$$

Now we replace $\left(w_{1}, \ldots, w_{n}\right)$ by $\zeta^{J^{*}}$, and since $\zeta^{n}=-1$, we get (1). For (2), replace $\left(z_{1}, \ldots, z_{n}\right)$ by $\left(\zeta^{i_{1}}, \ldots, \zeta^{i_{n}}\right)$. Then the formula comes from Lemma 6.1 (1). The 
formula (3) is a direct consequence of Lemma 6.1 (2). For (4), consider the following identity from Lemma 2.7 in [1]:

$$
\sum_{\lambda \in \mathcal{D}(n)} \widetilde{P}_{\lambda}\left(X_{n}^{w}\right) \widetilde{P}_{\hat{\lambda}}\left(X_{n}\right)=\left\{\begin{array}{cc}
S_{\rho_{n}}\left(X_{n}\right) & \text { if } w \in S_{n}, \\
0 & \text { if } w \in W_{n} \backslash S_{n}
\end{array}\right.
$$

where $w$ acts on $\left(x_{1}, \ldots, x_{n}\right)$ naturally; i.e., $s_{i}$ interchanges $x_{i}$ and $x_{i+1}$, and $\dot{s}_{n}$ interchanges $\pm x_{n}$ and $\mp x_{n+1}$. The formula (4) follows directly from the fact that for given $I, J \in \mathcal{I}_{n}$ with $I \neq J$, there is $w \in W_{n} \backslash S_{n}$ such that $\left(\zeta^{i_{1}}, \ldots, \zeta^{i_{n}}\right)^{w}=$ $\left(\zeta^{j_{1}}, \ldots, \zeta^{j_{n}}\right)$.

Remark. Let $A=\left[A_{I, \lambda}\right]$ be a square matrix defined by $A_{I, \lambda}=\widetilde{P}_{\lambda}\left(\zeta^{I}\right)$ for $I \in \mathcal{I}_{n}$ and $\lambda \in \mathcal{D}(n)$. Then it follows from (4) of Proposition 6.2 that the row vectors of $A$ are linearly independent, and so there is no zero row vector or zero column vector in $A$. In particular, there are nonzero entries in all rows and columns. Furthermore we have other kinds of orthogonality properties of $\widetilde{Q}$-polynomials (and hence $\widetilde{P}$ polynomials) given as follows.

Corollary 6.3. For any $\lambda, \mu \in \mathcal{D}(n)$, we have the following identities:

(1) $\sum_{I \in \mathcal{I}_{n}} \widetilde{P}_{\lambda}\left(\zeta^{I}\right) \widetilde{P}_{\hat{\mu}}\left(\zeta^{I}\right) \frac{1}{S_{\rho_{n}}\left(\zeta^{I}\right)}=\delta_{\lambda, \mu}$

(2) $\frac{1}{(2 n)^{n}} \sum_{I \in \mathcal{I}_{n}} P_{\lambda}\left(\zeta^{I^{*}}\right) \widetilde{Q}_{\mu}\left(\zeta^{I}\right)\left|\operatorname{Vand}\left(\zeta^{I}\right)\right|^{2}=\delta_{\lambda, \mu}$,

(3) $\frac{1}{(2 n)^{n}} \sum_{I \in \mathcal{I}_{n}} \overline{\omega\left(P_{\lambda}\right)\left(\zeta^{I^{*}}\right)} \widetilde{Q}_{\mu}\left(\zeta^{I}\right)\left|\operatorname{Vand}\left(\zeta^{I}\right)\right|^{2}=\delta_{\lambda, \mu}$.

Proof. First of all, we claim that $S_{\rho_{n}}\left(\zeta^{J}\right)$ cannot be zero. More generally, we show that $S_{\lambda}\left(\zeta^{J}\right)$ cannot be zero for any $\lambda \in \mathcal{R}(n)$ and any $J \in \mathcal{I}_{n}$, and if $\lambda, \mu$ are such that $|\lambda|=|\mu|$, then $S_{\lambda}\left(\zeta^{J}\right)$ and $S_{\mu}\left(\zeta^{J}\right)$ have the same parity. To prove this, first we note that $E_{1}\left(\zeta^{J}\right)=\zeta^{j_{1}}+\cdots+\zeta^{J_{n}} \neq 0$ for $J \in \mathcal{I}_{n}$, and $S_{\left(n^{n}\right)}\left(\zeta^{J}\right) \neq 0$ (Remark 4.5, [19]). Assume that $S_{\lambda}\left(\zeta^{J}\right)$ is not zero for any $\lambda$ with $|\lambda|=l$. Take $\mu \in \mathcal{R}(n)$ with $|\mu|=l-1$. Then, by the Pieri rule for Schur polynomials (3]), the multiplication can be written as

$$
S_{\mu}\left(\zeta^{J}\right) \cdot E_{1}\left(\zeta^{J}\right)=\sum S_{\lambda}\left(\zeta^{J}\right),
$$

where the sum is over all $\lambda$ with $\lambda \supset \mu$ and $|\lambda|=l$. By the induction hypothesis, $S_{\mu}\left(\zeta^{J}\right)$ cannot be zero and they have the same parity for all partitions with the same weight. For (1), consider the matrix $B=\left[B_{\mu, J}\right]$ defined by

$$
B_{\mu, J}:=\frac{\widetilde{P}_{\hat{\mu}}\left(\zeta^{J}\right)}{S_{\rho_{n}}\left(\zeta^{J}\right)}
$$

for $\mu \in \mathcal{D}(n)$ and $J \in \mathcal{I}_{n}$. Note that the entries of the multiplication $A B$ are given by the left hand side of (4) of Proposition 6.2. Therefore $A B$ is equal to the identity matrix, and so is the multiplication $B A:=\left[C_{\mu, \lambda}\right]$. The identity (1) follows since the left hand side of (1) is $C_{\mu, \lambda}$. The identities (2) and (3) follow by applying the same idea. 
6.2. Completion of proofs of Theorems 4.6, 4.8 and 4.10, To complete the proof of Theorem 4.6, we have to show that $\phi_{C}$ is injective. Let $F$ be an element of $\mathfrak{R}_{o}$ such that $\dot{F}=0$, i.e., $\dot{F}(u)=0$ for all $u \in \mathcal{V}_{n}^{C}$. Since the Schubert classes form a basis of $\Re_{o}=q H_{\mathbb{C}}^{*}\left(O G^{o}(n)\right)$ over $\mathbb{C}[q], F$ can be written as $F=\sum_{\lambda \in \mathcal{D}(n)} F_{\lambda} \widetilde{P}_{\lambda}$, where $F_{\lambda} \in \mathbb{C}[q]$. Then for any $t \in \mathbb{C}$ and $I \in \mathcal{I}_{n}$, we have

$$
0=\dot{F}\left(u_{2 n}\left(t \zeta^{I}\right)\right)=\sum_{\lambda \in \mathcal{D}(n)} F_{\lambda}\left(u_{2 n}\left(t \zeta^{I}\right)\right) \dot{\widetilde{P}}_{\lambda}\left(u_{2 n}\left(t \zeta^{I}\right)\right)=0
$$

and hence

$$
0=F\left(t \zeta^{I}\right)=\sum_{\lambda \in \mathcal{D}(n)} F_{\lambda}\left(t^{2 n}\right) \widetilde{P}_{\lambda}\left(t \zeta^{I}\right)=\sum_{\lambda \in \mathcal{D}(n)} t^{l(\lambda)} F_{\lambda}\left(t^{2 n}\right) \widetilde{P}_{\lambda}\left(\zeta^{I}\right),
$$

where $F_{\lambda}\left(t^{2 n}\right)$ is a polynomial in $t^{2 n}$. Therefore for fixed $t$, the vector $\left(t^{l(\lambda)} F_{\lambda}\left(t^{2 n}\right)\right)_{\lambda}$ lies in the orthogonal component of the subspace of $\mathbb{C}^{|\mathcal{D}(n)|}$ spanned by the vectors $\left(\widetilde{P}_{\lambda}\left(\zeta^{I}\right)\right)_{\lambda}$ indexed by $I \in \mathcal{I}_{n}$. But from the remark subsequent to Proposition 6.2. the vectors $\left(\widetilde{P}_{\lambda}\left(\zeta^{I}\right)\right)_{\lambda \in \mathcal{D}(n)}$ indexed by $I \in \mathcal{I}_{n}$ are linearly independent, and since $|\mathcal{D}(n)|=\left|\mathcal{I}_{n}\right|$, they form a basis for $\mathbb{C}^{|\mathcal{D}(n)|}$. Therefore the vector $\left(t^{l(\lambda)} F_{\lambda}\left(t^{2 n}\right)\right)_{\lambda}$ is a zero vector. Since this holds for all $t \in \mathbb{C}, F_{\lambda}$ is 0 in $\mathbb{C}[q]$ for all $\lambda \in \mathcal{D}(n)$. Therefore $\phi_{C}$ is injective. The same argument applies to the cases and $O G^{e}(n)$ and $L G(n)$ to complete the proof of Theorems 4.10 and 4.8 .

\subsection{Positivity of $\tilde{Q}$ - and $\tilde{P}$-polynomials.}

Lemma 6.4 (Perron-Frobenius Theorem). Suppose a matrix $A$ is nonnegative, i.e., all entries $A_{i, j}$ are nonnegative. Then there is a nonnegative eigenvalue $K$ with a nonnegative eigenvector and such that $K$ is maximal among absolute values of all eigenvalues of $A$.

Proof. We refer to [15] for a proof.

Lemma 6.5. For any $I \in \mathcal{I}_{n}$, we have $\widetilde{P}_{\rho_{n}}\left(\zeta^{I}\right) \neq 0$.

Proof. From Corollary 7 of [10], we have that $\tau_{\rho_{n}} \cdot \tau_{\rho_{n}}=\tau_{n} q^{n / 2}$ if $n$ is even, and $\tau_{\rho_{n}} \cdot \tau_{\rho_{n}}=q^{(n+1) / 2}$ if $n$ is odd. From the identification of $\tau_{\lambda}$ and $\widetilde{P}_{\lambda}$, and the fact (Lemma 5.5) that $\widetilde{P}_{n}\left(\zeta^{I}\right)$ is nonzero for any $I \in \mathcal{I}_{n}$, it follows that $\widetilde{P}_{\rho_{n}}\left(\zeta^{I}\right)$ is nonzero.

Theorem 6.6. We have the following inequalities.

(1) $\widetilde{P}_{\lambda}\left(\zeta^{I_{0}}\right)>0$ for all strict partitions $\lambda \in \mathcal{D}(n)$, and for $I \in \mathcal{I}_{n}^{s}$ with $I \neq I_{0}$, there exist strict partitions $\mu \in \mathcal{D}(n)$ such that $\widetilde{P}_{\mu}\left(\zeta^{I}\right)<0$. Furthermore, if $I \in \mathcal{I}_{n}$ is such that $\widetilde{P}_{\lambda}\left(\zeta^{I}\right) \geq 0$ for all $\lambda \in \mathcal{D}(n)$, then $I=I_{0}$.

(2) For any $\lambda \in \mathcal{D}(n), \widetilde{P}_{\lambda}\left(\zeta^{I_{0}}\right) \geq\left|\widetilde{P}_{\lambda}\left(\zeta^{I}\right)\right|$ for all $I \in \mathcal{I}_{n}$.

Proof. The idea of the proof will be taken more or less verbatim from Proposition 11.1 of [19, where the analogue of $(2)$ was obtained. Consider $q H_{\mathbb{C}}^{*}\left(O G^{o}(n)\right)_{q=1}:=$ $q H_{\mathbb{C}}^{*}\left(O G^{o}(n)\right) \otimes \mathbb{C}[q] /(q-1)$, the specialization of the quantum cohomology ring at $q=1$. By the isomorphism of Theorem 4.6, this ring may be viewed as the coordinate ring of the zero-dimensional subvariety $\left\{u_{2 n}\left(\zeta^{I}\right) \mid I \in \mathcal{I}_{n}\right\}$ of $\mathcal{V}_{n}^{C}$ cut out by the function $\dot{q}-1 \in \mathcal{O}\left(\mathcal{V}_{n}^{C}\right)$ and has a basis given by the Schubert basis $\tau_{\lambda}$, 
$\lambda \in \mathcal{D}(n)$. For $I \in \mathcal{I}_{n}$, define $\tau_{I}:=\sum_{\nu \in \mathcal{D}(n)} \widetilde{P}_{\nu}\left(\zeta^{I}\right) \tau_{\hat{\nu}}$. Then, by Proposition 6.2 (4), we have that

$$
\tau_{I}\left(u_{2 n}\left(\zeta^{J}\right)\right)=\delta_{I, J} S_{\rho_{n}}\left(\zeta^{J}\right)
$$

Therefore the vectors $\tau_{I}, I \in \mathcal{I}_{n}$, are linearly independent, and hence form a basis for $q H_{\mathbb{C}}^{*}\left(O G^{o}(n)\right)_{q=1}$. Define the multiplication operator $\left[\tau_{\lambda}\right]: \tau \mapsto \tau_{\lambda} \cdot \tau$ on $q H_{\mathbb{C}}^{*}\left(O G^{o}(n)\right)_{q=1}$. Then for each $I \in \mathcal{I}_{n}$, we have $\left[\tau_{\lambda}\right]\left(\tau_{I}\right)=\widetilde{P}_{\lambda}\left(\zeta^{I}\right) \tau_{I}$ for all $I \in \mathcal{I}_{n}$. So $\left[\tau_{\lambda}\right]$ has eigenvalues $\widetilde{P}_{\lambda}\left(\zeta^{I}\right), I \in \mathcal{I}_{n}$, with eigenvectors $\tau_{I}$. In fact the set $\left\{\tau_{I} \mid I \in \mathcal{I}_{n}\right\}$ is a simultaneous eigenbasis for the operators $[\tau]$ with $\tau \in q H_{\mathbb{C}}^{*}\left(O G^{o}(n)\right)_{q=1}$. Let $S$ be the set of elements $\tau$ in $q H_{\mathbb{R}}^{*}\left(O G^{o}(n)\right)_{q=1}$ such that $[\tau]$ has simple eigenvalues. Then $S$ is an open dense subset of $q H_{\mathbb{R}}^{*}\left(O G^{o}(n)\right)_{q=1}$, and so all $\left[\tau_{\lambda}\right]$ can be approximated to arbitrary precision by the operators $[\tau]$ with $\tau \in S$; for any small $\epsilon>0$, there are $0<\epsilon_{\mu}<\epsilon$ such that $\left[\tau_{\lambda}\right]+\sum_{\mu \in \mathcal{D}(n)} \epsilon_{\mu}\left[\tau_{\mu}\right]$ has simple eigenvalues.

Let $A_{\lambda}$ be the matrix of the operator $\left[\tau_{\lambda}\right]$ with respect to the basis $\left\{\tau_{\nu} \mid \nu \in\right.$ $\mathcal{D}(n)\}$. Since the entries $A_{\mu, \nu}^{\lambda}$ are Gromov-Witten invariants, $A_{\lambda}$ is nonnegative and so is the matrix $A_{\lambda}^{\epsilon}:=A_{\lambda}+\sum_{\mu} \epsilon_{\mu} A_{\mu}$. We also note that the vector $\tau_{I}$ has the coordinates $\left(\widetilde{P}_{\lambda}\left(\zeta^{I}\right)\right)_{\lambda \in \mathcal{D}(n)}$ with respect to the basis $\left\{\tau_{\nu} \mid \nu \in \mathcal{D}(n)\right\}$. First consider the case $\lambda=(1)$. In this case, $A_{(1)}$ has $\left|\mathcal{I}_{n}\right|$-distinct eigenvalues $\widetilde{P}_{1}\left(\zeta^{I}\right)$, $I \in \mathcal{I}_{n}$, with eigenvectors $\left(\widetilde{P}_{\lambda}\left(\zeta^{I}\right)\right)_{\lambda \in \mathcal{D}(n)}$. Therefore all the eigenvalues of $A_{(1)}$ are simple, and, by the Perron-Frobenius theorem, we have the maximum eigenvalue $\widetilde{P}_{1}\left(\zeta^{I_{0}}\right)=\frac{1}{2} E_{1}\left(\zeta^{I_{0}}\right)$ with a nonnegative eigenvector, which is $\left(\widetilde{P}_{\lambda}\left(\zeta^{I_{0}}\right)\right)_{\lambda \in \mathcal{D}(n)}$ or $-\left(\widetilde{P}_{\lambda}\left(\zeta^{I_{0}}\right)\right)_{\lambda \in \mathcal{D}(n)}$. But the vector $-\left(\widetilde{P}_{\lambda}\left(\zeta^{I_{0}}\right)\right)_{\lambda \in \mathcal{D}(n)}$ cannot be nonnegative since $\widetilde{P}_{1}\left(\zeta^{I_{0}}\right)>0$. Therefore $\left(\widetilde{P}_{\lambda}\left(\zeta^{I_{0}}\right)\right)_{\lambda \in \mathcal{D}(n)}$ is a nonnegative eigenvector, and so $\widetilde{P}_{\lambda}\left(\zeta^{I_{0}}\right) \geq 0$ for all $\lambda \in \mathcal{D}(n)$. For the second statement of $(1)$, fix $I \in \mathcal{I}_{n}$ with $I \neq I_{0}$. Since $\widetilde{P}_{\rho_{n}}\left(\zeta^{I}\right)$ is nonzero by Lemma 6.5. Proposition 6.2 (4) implies that there is a strict partition $\mu \in \mathcal{D}(n)$ such that $\widetilde{P}_{\mu}\left(\zeta^{I}\right)<0$. This proves the second statement of (1). The last statement of (1) follows from the second statement of (1) and the fact that $I \in \mathcal{I}_{n}^{s}$ if and only if $\widetilde{P}_{\lambda}\left(\zeta^{I}\right)$ are real for all $\lambda \in \mathcal{D}(n)$. This proves (1), except that $\widetilde{P}_{\lambda}\left(\zeta^{I_{0}}\right)$ are strictly positive for all $\lambda \in \mathcal{D}(n)$. This will be done after proving (2). For (2), we apply the Perron-Frobenius Theorem above to $A_{\lambda}^{\epsilon}$. Then there is $I \in \mathcal{I}_{n}$ such that

$$
K_{\lambda}^{\epsilon}(I):=\widetilde{P}_{\lambda}\left(\zeta^{I}\right)+\sum_{\mu} \epsilon_{\mu} \widetilde{P}_{\mu}\left(\zeta^{I}\right)
$$

is a maximal real eigenvalue with a nonnegative eigenvector, and such that $K_{\lambda}^{\epsilon}(I) \geq$ $\left|K_{\lambda}^{\epsilon}(J)\right|$ for all $J \in \mathcal{I}_{n}$, But since the vector $\left(\widetilde{P}_{\mu}\left(\zeta^{I_{0}}\right)\right)_{\mu \in \mathcal{D}(n)}$ is a unique nonnegative eigenvector (up to a positive scalar) by the last statement of (1), we have $I=I_{0}$, and for all $I \in \mathcal{I}_{n}$,

$$
K_{\lambda}^{\epsilon}\left(I_{0}\right) \geq\left|K_{\lambda}^{\epsilon}(I)\right|
$$

Since this is true for all $\epsilon>0$, we have $\widetilde{P}_{\lambda}\left(\zeta^{I_{0}}\right) \geq\left|\widetilde{P}_{\lambda}\left(\zeta^{I}\right)\right|$ for all $I \in \mathcal{I}_{n}$. This proves (2). Now we prove that $\widetilde{P}_{\lambda}\left(\zeta^{I_{0}}\right)$ are strictly positive for all $\lambda \in \mathcal{D}(n)$. If $\widetilde{P}_{\lambda}\left(\zeta^{I_{0}}\right)$ is zero for some $\lambda \in \mathcal{D}(n)$, by $(2), \widetilde{P}_{\lambda}\left(\zeta^{I}\right)$ is zero for all $I \in \mathcal{I}_{n}$, which is impossible by the remark subsequent to Proposition 6.2. Therefore $\widetilde{P}_{\lambda}\left(\zeta^{I_{0}}\right)$ is strictly positive for all $\lambda \in \mathcal{D}(n)$. This completes the proof. 


\section{Gromov-Witten invariants}

In this section, we use the orthogonality formulas given in Section [6] and Theorems 4.6 and 4.8 to derive the Vafa-Intriligator type formulas, which compute the 3-point, genus zero, Gromov-Witten invariants for $O G^{o}(n)\left(O G^{e}(n)\right)$ and $L G(n)$. We start with the following preliminary results, which will be crucial to find the Vafa-Intriligator type formulas.

Lemma 7.1. Let $I \in \mathcal{I}_{n}, t \in \mathbb{C}^{*}, P$ be a homogeneous symmetric polynomial in the variables $x_{1}, \ldots, x_{n}$. Then we have the following expressions:

$$
P\left(t \zeta^{I}\right)=\sum_{\nu \in \mathcal{R}(n)} m_{\nu}^{P}(t) \widetilde{Q}_{\nu}\left(t \zeta^{I}\right),
$$

where $m_{\nu}^{P}(t)=\frac{1}{(2 n)^{n}} \sum_{J \in \mathcal{I}_{n}} P\left(t \zeta^{J}\right) P_{\nu}\left(t^{-1} \zeta^{J^{*}}\right)\left|\operatorname{Vand}\left(\zeta^{J}\right)\right|^{2}$.

Proof. By Proposition 6.2 (2), we have the expression for $P\left(t \zeta^{I}\right)$ :

$$
P\left(t \zeta^{I}\right)=\frac{1}{(2 n)^{n}} \sum_{J \in \mathcal{I}_{n}} P\left(t \zeta^{J}\right)\left|\operatorname{Vand}\left(\zeta^{J}\right)\right|^{2} \sum_{\nu \in \mathcal{R}(n)} P_{\nu}\left(\zeta^{J^{*}}\right) \widetilde{Q}_{\nu}\left(\zeta^{I}\right)
$$

which is equal to

$$
\frac{1}{(2 n)^{n}} \sum_{\nu \in \mathcal{R}(n)} \sum_{J \in \mathcal{I}_{n}} P\left(t \zeta^{J}\right)\left|\operatorname{Vand}\left(\zeta^{J}\right)\right|^{2} P_{\nu}\left(t^{-1} \zeta^{J^{*}}\right) \widetilde{Q}_{\nu}\left(t \zeta^{I}\right) .
$$

This proves the lemma.

Lemma 7.2 ([19]). Let $m$ be a homogeneous polynomial in $n$ variables. Then we have $\sum_{J \in \mathcal{I}_{n}} m\left(\zeta^{J}\right)=0$ unless $\operatorname{deg}(m) \equiv 0 \bmod 2 n$. In particular,

$$
m_{\nu}^{P}(t)=\frac{1}{(2 n)^{n}} t^{(\operatorname{deg} P-|\nu|)} \sum_{J \in \mathcal{I}_{n}} P\left(\zeta^{J}\right) P_{\nu}\left(\zeta^{J^{*}}\right)\left|\operatorname{Vand}\left(\zeta^{J}\right)\right|^{2}
$$

if $\operatorname{deg} P \equiv|\nu| \bmod 2 n$, and otherwise $m_{\nu}^{P}(t)=0$.

Proof. Let $M(t):=\sum_{J \in \mathcal{I}_{n}} m\left(t \zeta^{J}\right)$. Then we have the relation $M(t)=M(t \zeta)$, and hence $M(t)$ is a polynomial in $t^{2 n}$. Therefore unless $\operatorname{deg} m$ is divisible by $2 n$, we must have $M(t)=t^{\operatorname{deg} m} \sum_{J \in \mathcal{I}_{n}} m\left(\zeta^{J}\right)=0$ for all $t$. It follows that $\sum_{J \in \mathcal{I}_{n}} m\left(t \zeta^{J}\right)=0$ unless $\operatorname{deg} m$ is divisible by $2 n$. Apply the same analysis to $m_{\nu}^{P}(t)$ to get the rest of the lemma.

\subsection{Vafa-Intriligator type formula for $O G^{o}(n)\left(O G^{e}(n)\right)$.}

Proposition 7.3. Let $X_{i, i}$ be given as in (4.9), $\Im_{C}$ the ideal of $\mathbb{Z}\left[X_{1}, \ldots, X_{n}\right]$ generated by $X_{i, i}, i=1, \ldots, n-1$, and $\mathfrak{R}_{C}^{\mathbb{Z}}:=\mathbb{Z}\left[X_{1}, \ldots, X_{n}\right] / \mathfrak{I}_{C}$. If $\dot{P}$ is an element of $\mathfrak{R}_{C}^{\mathbb{Z}} \subset \mathcal{O}\left(\mathcal{V}_{n}^{C}\right)$, then $\dot{P}$ can be expressed as

$$
\dot{P}=\sum_{\nu \in \mathcal{D}(n), k \in \mathbb{Z}_{\geq 0}} \dot{P}_{\nu, k} \dot{q}^{k} \dot{\widetilde{P}}_{\nu}
$$


Here $\dot{P}_{\nu, k}$ is an integer defined as follows. If $\nu$ is a strict partition such that $\operatorname{deg} \dot{P}=|\nu|+2 n k$ for some nonnegative integer $k$, then

$$
\dot{P}_{\nu, k}:=\frac{2^{l(\nu)+2 k}}{(2 n)^{n}} \sum_{m=0}^{a(\nu)} \sum_{J \in \mathcal{I}_{n}} P\left(\zeta^{J}\right) P_{\nu(m)}\left(\zeta^{J^{*}}\right)\left|\operatorname{Vand}\left(\zeta^{J}\right)\right|^{2},
$$

and otherwise $\dot{P}_{\nu, k}:=0$, where $a(\nu)=\left\lfloor\frac{n-l(\nu)}{2}\right\rfloor$, and $\nu(m)=\left((n)^{(2 m)}, \nu_{1}, \ldots, \nu_{l}\right)$.

Proof. Note that $\mathfrak{R}_{C}^{\mathbb{Z}}$ is the image of $q H^{*}\left(O G^{o}(n)\right)$ under the isomorphism of Theorem 4.6. Therefore the set $\left\{\dot{\widetilde{P}}_{\nu} \dot{q}^{k} \mid k \in \mathbb{Z}_{\geq 0}, \nu \in \mathcal{D}(n)\right\}$ is a $\mathbb{Z}$-basis for the ring $\mathfrak{R}_{C}^{\mathbb{Z}}$, and so $\dot{P}_{\nu, k}$ are integers. To get (7.26) , we consider the following expression for $P\left(t \zeta^{I}\right)$ from Lemma 7.1:

$$
P\left(t \zeta^{I}\right)=\sum_{\nu \in \mathcal{R}(n)} \sum_{J \in \mathcal{I}_{n}} \frac{\left|\operatorname{Vand}\left(\zeta^{J}\right)\right|^{2}}{(2 n)^{n}} P\left(\zeta^{J}\right) P_{\nu}\left(\zeta^{J^{*}}\right) t^{(\operatorname{deg} P-|\nu|)} \widetilde{Q}_{\nu}\left(t \zeta^{I}\right) .
$$

By Lemma 7.2, the terms in (7.27) corresponding to $\nu$ with $2 n \nmid(\operatorname{deg} P-|\nu|)$ vanish, and so $P\left(t \zeta^{I}\right)$ can be written as

$$
P\left(t \zeta^{I}\right)=\sum_{\nu \in A} \sum_{J \in \mathcal{I}_{n}} \frac{\left|\operatorname{Vand}\left(\zeta^{J}\right)\right|^{2}}{(2 n)^{n}} P\left(\zeta^{J}\right) P_{\nu}\left(\zeta^{J^{*}}\right) t^{(\operatorname{deg} P-|\nu|)} \widetilde{Q}_{\nu}\left(t \zeta^{I}\right),
$$

where the index set $A$ is the set of all partitions $\nu \in \mathcal{R}(n)$ such that $\operatorname{deg} P-|\nu|$ is divisible by $2 n$.

If we evaluate the identity of functions on $\mathcal{V}_{n}^{C}$ determined by this identity, then the terms $\dot{\widetilde{Q}}_{\nu}$ vanish for nonstrict partitions $\nu$ with repeated parts from $\{1,2, \ldots, n-1\}$, by the fact that $\dot{\widetilde{Q}}_{i, i}=0$ in the ring $\mathcal{O}\left(\mathcal{V}_{n}^{C}\right)$ for $i=1, \ldots, n-1$, and by the factorization property of $\widetilde{Q}$-polynomials (2) in $\$ 2.5)$. Therefore, for $u\left(t \zeta^{I}\right)=u_{2 n}\left(t \zeta^{I}\right) \in \mathcal{V}_{n}^{C}$, we have

$$
\dot{P}\left(u\left(t \zeta^{I}\right)\right)=\sum_{\nu \in B} \sum_{J \in \mathcal{I}_{n}} \frac{\left|\operatorname{Vand}\left(\zeta^{J}\right)\right|^{2}}{(2 n)^{n}} \dot{P}\left(u\left(\zeta^{J}\right)\right) \dot{P}_{\nu}\left(u\left(\zeta^{J^{*}}\right)\right) t^{(\operatorname{deg} P-|\nu|)} \dot{\widetilde{Q}}_{\nu}\left(u\left(t \zeta^{I}\right)\right),
$$

where $B$ is the subset of $A$ consisting of $\nu$ such that $\nu$ has no repetitions from $\{1, \ldots, n-1\}$. Note that each partition in $B$ is of the form $\nu(m)$ for some strict partition $\nu \in \mathcal{D}(n)$ and a nonnegative integer $m$. Using the factorization property of $\widetilde{Q}$-polynomials ((2) in $\$ 2.5)$, and the identities $\widetilde{Q}_{\left(n^{2 m}\right)}=\widetilde{Q}_{\left(n^{2}\right)}^{m}, \dot{\widetilde{Q}}_{\left(n^{2}\right)}\left(u\left(t \zeta^{I}\right)\right)=t^{2 n}$, we can rewrite

$$
\begin{aligned}
& \dot{P}\left(u\left(t \zeta^{I}\right)\right) \\
& =\sum_{\nu \in \mathcal{D}(n)} \sum_{m=0}^{a(\nu)} \sum_{J \in \mathcal{I}_{n}} \frac{\left|\operatorname{Vand}\left(\zeta^{J}\right)\right|^{2}}{(2 n)^{n}} \dot{P}\left(u\left(\zeta^{J}\right)\right) \dot{P}_{\nu(m)}\left(u\left(\zeta^{J^{*}}\right)\right) t^{(\operatorname{deg} P-|\nu(m)|)} \dot{\widetilde{Q}}_{\nu(m)}\left(u\left(t \zeta^{I}\right)\right) \\
& =\sum_{\nu \in \mathcal{D}(n)} \sum_{m=0}^{a(\nu)} \sum_{J \in \mathcal{I}_{n}} \frac{\left|\operatorname{Vand}\left(\zeta^{J}\right)\right|^{2}}{(2 n)^{n}} \dot{P}\left(u\left(\zeta^{J}\right)\right) \dot{P}_{\nu(m)}\left(u\left(\zeta^{J^{*}}\right)\right) \\
& \quad \quad \times t^{(\operatorname{deg} P-|\nu(m)|)} \dot{\widetilde{Q}}_{\nu}\left(u\left(t \zeta^{I}\right)\right) \dot{\widetilde{Q}}_{\left(n^{2 m}\right)}\left(u\left(t \zeta^{I}\right)\right) \\
& =\sum_{\nu \in \mathcal{D}(n)} \sum_{m=0}^{a(\nu)} \sum_{J \in \mathcal{I}_{n}} \frac{\left|\operatorname{Vand}\left(\zeta^{J}\right)\right|^{2}}{(2 n)^{n}} P\left(\zeta^{J}\right) P_{\nu(m)}\left(\zeta^{J^{*}}\right) \dot{\widetilde{Q}}_{\nu}\left(u\left(t \zeta^{I}\right)\right) \dot{\widetilde{Q}}_{\left(n^{2}\right)}\left(u\left(t \zeta^{I}\right)\right)
\end{aligned}
$$


Here the upper bound $a(\nu)$ is taken since $\nu(m)$ belongs to $\mathcal{R}(n)$.

Since $u\left(t \zeta^{I}\right)$ are arbitrary points in $\mathcal{V}_{n}^{C}, \dot{\widetilde{Q}}_{\nu}=2^{l(\nu)} \dot{\widetilde{P}}_{\nu}$ and $\dot{\widetilde{Q}}_{\left(n^{2}\right)}=4 \dot{q}$, the function $\dot{P}$ can be written as in (7.25), with $\dot{P}_{\nu, k}$ defined as in (7.26).

Corollary 7.4. For partitions $\lambda, \mu, \nu \in \mathcal{D}(n)$, and a nonnegative integer $k$, the Gromov-Witten invariant $\left\langle\tau_{\lambda}, \tau_{\mu}, \tau_{\hat{\nu}}\right\rangle_{k}$ for $O G^{o}(n)\left(\cong O G^{e}(n)\right)$ is given by

$$
\left\langle\tau_{\lambda}, \tau_{\mu}, \tau_{\hat{\nu}}\right\rangle_{k}=\frac{2^{l(\nu)+2 k}}{(2 n)^{n}} \sum_{m=0}^{a(\nu)} \sum_{J \in \mathcal{I}_{n}} \widetilde{P}_{\lambda}\left(\zeta^{J}\right) \widetilde{P}_{\mu}\left(\zeta^{J}\right) P_{\nu(m)}\left(\zeta^{J^{*}}\right)\left|\operatorname{Vand}\left(\zeta^{J}\right)\right|^{2},
$$

whenever $|\lambda|+|\mu|=|\nu|+2 n k$, and otherwise by $\left\langle\tau_{\lambda}, \tau_{\mu}, \tau_{\hat{\nu}}\right\rangle_{k}=0$.

Proof. Apply Proposition 7.3 to $\dot{P}=\dot{\widetilde{P}}_{\lambda} \dot{\widetilde{P}}_{\mu}$. Then the Gromov-Witten invariant $\left\langle\tau_{\lambda}, \tau_{\mu}, \tau_{\hat{\nu}}\right\rangle_{k}$ is the coefficient of $\dot{\widetilde{P}}_{\nu} \dot{q}^{k}$ in the expansion of $\dot{\widetilde{P}}_{\lambda} \dot{\widetilde{P}}_{\mu}$ in the basis $\left\{\widetilde{P}_{\nu} \dot{q}^{k}\right.$ $\nu \in \mathcal{D}(n)$ and $k \geq 0\}$, which is given as above.

\subsection{Vafa-Intriligator type formula for $L G(n)$.}

Proposition 7.5. Let $X_{i, i}$ be given as in (4.9), $\mathfrak{I}_{B}$ the ideal of $\mathbb{Z}\left[X_{1}, \ldots, X_{n+1}\right]$ generated by $X_{i, i}, i=1, \ldots, n$, and $\mathfrak{R}_{B}^{\mathbb{Z}}:=\mathbb{Z}\left[X_{1}, \ldots, X_{n+1}\right] / \mathfrak{I}_{B}$. If $\dot{P}$ is an element of $\mathfrak{R}_{B}^{\mathbb{Z}} \subset \mathcal{O}\left(\mathcal{V}_{n}^{B}\right)$, then $\dot{P}$ can be expressed as

$$
\dot{P}=\sum_{\nu \in \mathcal{D}(n), d \in \mathbb{Z}_{\geq 0}} \dot{P}_{\nu, d} \dot{q}^{d} \dot{\widetilde{Q}}_{\nu}
$$

Here $\dot{P}_{\nu, d}$ is an integer defined as follows. If $\nu$ is a strict partition in $\mathcal{D}(n)$ with $\operatorname{deg} \dot{P}=|\nu|+(n+1) d$ for some nonnegative integer $d$, then

$$
\dot{P}_{\nu, d}:=\frac{1}{2^{d}(2 n+2)^{(n+1)}} \sum_{m=0}^{b(\nu)} \sum_{J \in \mathcal{I}_{n+1}} P\left(\zeta^{J}\right) P_{\nu[m]}\left(\zeta^{J^{*}}\right)\left|\operatorname{Vand}\left(\zeta^{J}\right)\right|^{2} .
$$

and otherwise $\dot{P}_{\nu, d}:=0$, where $\nu[m]$ and $b(\nu)$ are defined as

$$
\begin{aligned}
\nu[m]: & = \begin{cases}\left((n+1)^{2 m}, \nu_{1}, \ldots \nu_{l}\right) & \text { if } d \text { is even, } \\
\left((n+1)^{2 m+1}, \nu_{1}, \ldots, \nu_{l}\right) & \text { if } d \text { is odd, }\end{cases} \\
b(\nu): & = \begin{cases}\left\lfloor\frac{n+1-l(\nu)}{\left.L_{(\nu)}^{2}\right\rfloor}\right. & \text { if } d \text { is even, } \\
\left\lfloor\frac{n-l(\nu)}{2}\right\rfloor & \text { if } d \text { is odd. }\end{cases}
\end{aligned}
$$

Proof. Note that $\mathfrak{R}_{B}^{\mathbb{Z}}$ is the image of $H^{*}(L G(n))$ under the isomorphism of Theorem 4.8. Therefore the set $\left\{\dot{\widetilde{Q}}_{\nu} \dot{q}^{d} \mid \nu \in \mathcal{D}(n), d \geq 0\right\}$ is a $\mathbb{Z}$-basis for the ring $\mathfrak{R}_{B}^{\mathbb{Z}}$, and so $\dot{P}_{\nu, d}$ are integers. For (7.30), we consider the following expression for $\dot{P}$ from Lemma 7.1

$$
\dot{P}\left(u\left(t \zeta^{I}\right)\right)=\sum_{\nu \in \mathcal{R}(n+1)} \sum_{J \in \mathcal{I}_{n+1}} \frac{\left|\operatorname{Vand}\left(\zeta^{J}\right)\right|^{2}}{(2 n+2)^{(n+1)}} \dot{P}\left(u\left(t \zeta^{J}\right)\right) \dot{P}_{\nu}\left(u\left(t^{-1} \zeta^{J^{*}}\right)\right) \dot{\widetilde{Q}}_{\nu}\left(u\left(t \zeta^{I}\right)\right),
$$

where $u\left(t \zeta^{I}\right)=u_{2 n+1}\left(t \zeta^{I}\right) \in \mathcal{V}_{n}^{B}$.

If we apply Lemma 7.2 to the above identity, the terms $\dot{\widetilde{Q}}_{\nu}$ vanish for the partitions $\nu$ with $(2 n+2) \nmid(\operatorname{deg} P-|\nu|)$, and if we evaluate $\dot{P}$ on the points of $\mathcal{V}_{n}^{B}$, then the terms $\dot{\widetilde{Q}}_{\nu}$ vanish for nonstrict partitions $\nu$ with repeated parts from $\{1,2, \ldots, n\}$, 
since the variety $\mathcal{V}_{n}^{B}$ is defined by the equations $\dot{\widetilde{Q}}_{i, i}=0$ for $i=1, \ldots, n$. Therefore $\dot{P}\left(t \zeta^{I}\right)$ is reduced to the following expression:

$$
\sum_{\nu \in A} \sum_{J \in \mathcal{I}_{n+1}} \frac{\left|\operatorname{Vand}\left(\zeta^{J}\right)\right|^{2}}{(2 n+2)^{n+1}} \dot{P}\left(u\left(t \zeta^{J}\right)\right) \dot{P}_{\nu}\left(u\left(t^{-1} \zeta^{J^{*}}\right)\right) \dot{\widetilde{Q}}_{\nu}\left(u\left(t \zeta^{I}\right)\right)
$$

where the index set $A$ is the set of all partitions $\nu=\left(\nu_{1}, \ldots, \nu_{l}\right) \in \mathcal{R}(n+1)$ such that if $\nu_{i} \leq n$ for some $i$, then $\nu_{i}>\nu_{i+1}>\cdots>\nu_{l}$, and such that $\operatorname{deg} P-|\nu|$ is divisible by $2(n+1)$. By applying the factorization property of $\widetilde{Q}$-polynomials ([3] in 92.5$)$ and regrouping the terms, we can write $\dot{P}\left(t \zeta^{I}\right)$ as follows:

$$
\begin{aligned}
& \sum_{\nu \in B} \sum_{m=0}^{b(\nu)} \sum_{J \in \mathcal{I}_{n+1}} \frac{\left|\operatorname{Vand}\left(\zeta^{J}\right)\right|^{2}}{(2 n+2)^{n+1}} \dot{P}\left(u\left(\zeta^{J}\right)\right) \dot{P}_{\nu[m]}\left(u\left(\zeta^{J^{*}}\right)\right) \\
& \times t^{(\operatorname{deg} P-|\nu(m)|)} \dot{\widetilde{Q}}_{\nu}\left(u\left(t \zeta^{I}\right)\right) \dot{\widetilde{Q}}_{\left((n+1)^{r}\right)}\left(u\left(t \zeta^{I}\right)\right),
\end{aligned}
$$

where the index set $B$ is the set of all partitions $\nu \in \mathcal{D}(n)$ such that $\operatorname{deg} P-|\nu|$ is divisible by $(n+1), r=2 m$ if $\frac{\operatorname{deg} P-|\nu|}{n+1}$ is even, $r=2 m+1$ otherwise, and the second sum is taken up to $b(\nu)$ since $\nu[m]$ belongs to $\mathcal{R}(n+1)$.

Using the relation $q=2 \widetilde{Q}_{n+1}$, we can rewrite $\dot{P}\left(t \zeta^{I}\right)$ as follows:

$$
\sum_{\nu \in B} \sum_{m=0}^{b(\nu)} \sum_{J \in \mathcal{I}_{n+1}} \frac{\left|\operatorname{Vand}\left(\zeta^{J}\right)\right|^{2}}{k(P, n, \nu)} \dot{P}\left(u\left(\zeta^{J}\right)\right) \dot{P}_{\nu[m]}\left(u\left(\zeta^{J^{*}}\right)\right) \dot{\widetilde{Q}}_{\nu}\left(u\left(t \zeta^{I}\right)\right) \dot{q}^{\frac{\operatorname{deg} P-|\nu|}{n+1}}\left(u\left(t \zeta^{I}\right)\right),
$$

where $k(P, n, \nu)=2^{\frac{\operatorname{deg} P-|\nu|}{n+1}}(2 n+2)^{n+1}$.

If $d$ is a nonnegative integer such that $\operatorname{deg} \dot{P}=|\nu|+(n+1) d$, and so $\frac{\operatorname{deg} P-|\nu|}{n+1}=d$, then the coefficient of $\dot{\widetilde{Q}}_{\nu}\left(u\left(t \zeta^{I}\right)\right) \dot{q}^{k}\left(u\left(t \zeta^{I}\right)\right)$ is $\dot{P}_{\nu, d}$, given as in (7.30).

Corollary 7.6. For partitions $\lambda, \mu$, and $\nu \in \mathcal{D}(n)$, and a nonnegative integer $d$, the Gromov-Witten invariant $\left\langle\sigma_{\lambda}, \sigma_{\mu}, \sigma_{\hat{\nu}}\right\rangle_{d}$ for $L G(n)$ is given by

$$
\left\langle\sigma_{\lambda}, \sigma_{\mu}, \sigma_{\hat{\nu}}\right\rangle_{d}=\frac{1}{2^{d}(2 n+2)^{(n+1)}} \sum_{m=0}^{b(\nu)} \sum_{J \in \mathcal{I}_{n+1}} \widetilde{Q}_{\lambda}\left(\zeta^{J}\right) \widetilde{Q}_{\mu}\left(\zeta^{J}\right) P_{\nu[m]}\left(\zeta^{J^{*}}\right)\left|\operatorname{Vand}\left(\zeta^{J}\right)\right|^{2},
$$

whenever $|\lambda|+|\mu|=|\nu|+(n+1) d$, and otherwise by $\left\langle\sigma_{\lambda}, \sigma_{\mu}, \sigma_{\hat{\nu}}\right\rangle_{d}=0$.

Proof. If we apply Proposition7.5 to $\dot{P}=\dot{\widetilde{Q}}_{\lambda} \dot{\widetilde{Q}}_{\mu}$, the right hand side of (7.31) is the coefficient of $\dot{\widetilde{P}}_{\nu} \dot{q}^{k}$ in the expansion of $\dot{\widetilde{P}}_{\lambda} \dot{\widetilde{P}}_{\mu}$ in the basis $\left\{\widetilde{P}_{\nu} \dot{q}^{d} \mid \nu \in \mathcal{D}(n)\right.$ and $d \geq$ $0\}$, which is the Gromov-Witten invariant $\left\langle\sigma_{\lambda}, \sigma_{\mu}, \sigma_{\hat{\nu}}\right\rangle_{d}$.

For a partition $\nu \in \mathcal{D}(n-1) \subset \mathcal{D}(n)$, let $\hat{\lambda}^{n}$ and $\hat{\lambda}^{(n-1)}$ be the partitions that complement $\lambda$ in the sets $\{1,2, \ldots, n\}$ and $\{1,2, \ldots, n-1\}$, respectively, and denote $\tilde{\nu}:=(n, \nu)$. We can relate the quantum cohomology rings $q H^{*}\left(O G^{e}(n)\right)$ and $q H^{*}(L G(n-1))$ to each other in the following way. 
Corollary $7.7([10])$. Let $\lambda, \mu$ and $\nu$ be partitions in $\mathcal{D}(n-1)$. Then we have the following identities:

$$
\begin{aligned}
& \left\langle\tau_{\lambda}, \tau_{\mu}, \tau_{\hat{\nu}^{n}}\right\rangle_{k}=2^{4 k+l(\nu)-l(\lambda)-l(\mu)}\left\langle\sigma_{\lambda}, \sigma_{\mu}, \sigma_{\hat{\nu}^{(n-1)}}\right\rangle_{2 k}, \\
& \left\langle\tau_{\lambda}, \tau_{\mu}, \tau_{\hat{\nu}^{(n-1)}}\right\rangle_{k}=2^{4 k+1+l(\tilde{\nu})-l(\lambda)-l(\mu)}\left\langle\sigma_{\lambda}, \sigma_{\mu}, \sigma_{\hat{\nu}^{(n-1)}}\right\rangle_{2 k+1} .
\end{aligned}
$$

Proof. These identities follow easily by comparing (7.29) and (7.31).

\section{Total POSITIVITy}

A matrix $A \in G L_{n}(\mathbb{R})$ is said to be totally positive (resp. totally nonnegative) if all the minors of $A$ are positive (resp. nonnegative). These matrices form a semi-algebraic subset of $G L_{n}(\mathbb{R})$. Total positivity for $G L_{n}(\mathbb{R})$ was mainly studied around the 1950s by Schoenberg, Gantmacher-Krein and others, and has diverse applications such as oscillating mechanical systems and planar Markov processes. Since it was generalized to all the reductive algebraic groups by Lusztig in the early 1990s [12, the total positivity has been more noted by its connections with the canonical bases. Furthermore, very recently it was shown by Rietsch that in type $A$, the total positivity of $\mathcal{V}_{P}$ has a very close connection with the positivity of Schubert basis functions on $\mathcal{V}_{P}$; more precisely, the set of the totally nonnegative elements in the varieties $\mathcal{V}_{P}$ coincides with the set where the Schubert basis functions are nonnegative ([19), and it was conjectured that this will be true for the other classical Lie types ([20]). In this section, we explicitly describe the totally positive parts of the varieties $\mathcal{V}_{n}^{C}$ and $\mathcal{V}_{n}^{B}$, and characterize them via the Schubert basis functions.

8.1. Total positivity of $U^{+}$and its Bruhat cells. Let $U^{+}(\mathbb{R})$ be the group of real points in $U^{+}$. For each $i=1, \ldots, n$, let $x_{i}(t):=\exp \left(t e_{i}\right)$ be the one-parameter subgroup corresponding to the simple root vector $e_{i}$. Define the subset $U^{+}\left(\mathbb{R}_{\geq 0}\right)$ of totally nonnegative elements in $U^{+}$as the multiplicative semigroup generated by all $x_{i}(t)$ with $i=1, \ldots, n$, and $t \geq 0$. For any $w \in W$, we set $U_{w}^{+}\left(\mathbb{R}_{>0}\right):=U^{+}\left(\mathbb{R}_{>0}\right) \cap$ $B^{-} w B^{-}$. Then since $G=\bigcup_{w \in W} B^{-} w B^{-}$, we have $U^{+}\left(\mathbb{R}_{\geq 0}\right)=\bigcup_{w \in W} U_{w}^{+}\left(\mathbb{R}_{>0}\right)$. The algebraic subset $U_{w_{0}}^{+}\left(\mathbb{R}_{>0}\right)$ is called the totally positive part of $U^{+}$, denoted by $U^{+}\left(\mathbb{R}_{>0}\right)$, and it is open in $U^{+}(\mathbb{R})$ and its closure is the set of all totally nonnegative elements in $U^{+}$, i.e., $\overline{U^{+}\left(\mathbb{R}_{>0}\right)}=U^{+}\left(\mathbb{R}_{\geq 0}\right)$. If $G$ is $G L_{n}(\mathbb{C})$, the above definitions coincide with the classical ones. In case $G$ is $S p_{2 n}(\mathbb{C})\left(\right.$ resp. $\left.S O_{2 n+1}(\mathbb{C})\right)$, the total positivity of $\mathrm{G}$ can be understood via that of $\widetilde{G}:=S L_{2 n}(\mathbb{C})\left(\right.$ resp. $S L_{2 n+1}(\mathbb{C})$ ). With the matrices $J$ in $\S \$ 2.2$ and 2.3 for $S p_{2 n}(\mathbb{C})$ and $S O_{2 n+1}(\mathbb{C})$, respectively, the group $G$ is naturally embedded into $\widetilde{G}$. More precisely, we have

$$
U^{+}=\widetilde{U}^{+} \cap G \quad \text { and } \quad T=\widetilde{T} \cap G,
$$

where $\widetilde{U}^{+}$and $\widetilde{T}$ are unipotent upper triangular matrices and diagonal matrices of $S L_{2 n}(\mathbb{C})$ or $S L_{2 n+1}(\mathbb{C})$. Furthermore, this embedding preserves the total positivity of $U^{+}$and Bruhat cells of $U^{+}([2])$; that is, for each $w \in W$,

$$
U_{w}^{+}\left(\mathbb{R}_{>0}\right)=\widetilde{U}_{\tilde{w}}^{+}\left(\mathbb{R}_{>0}\right) \cap G \text { and } U^{+}\left(\mathbb{R}_{\geq 0}\right)=\widetilde{U}^{+}\left(\mathbb{R}_{\geq 0}\right) \cap G,
$$

where $\tilde{w}$ is the image of $w$ under a proper embedding ([2]) of $W$ into $\widetilde{W}=S_{2 n}$.

Definition. For each parabolic subgroup $P \subset G$, denote by $\mathcal{V}_{P}(\mathbb{R})$ the set of all the real points of $\mathcal{V}_{P} \subset G^{\vee}$. Define

$$
\mathcal{V}_{P}\left(\mathbb{R}_{\geq 0}\right):=\mathcal{V}_{P}(\mathbb{R}) \cap\left(U^{\vee}\right)^{+}\left(\mathbb{R}_{\geq 0}\right) \text { and } \mathcal{V}_{P}\left(\mathbb{R}_{>0}\right):=\mathcal{V}_{P}\left(\mathbb{R}_{\geq 0}\right) \cap\left(B^{\vee}\right)^{-} w^{P}\left(B^{\vee}\right)^{-} \text {. }
$$


That is, $\mathcal{V}_{P}\left(\mathbb{R}_{\geq 0}\right)$ is the set of totally nonnegative elements in $\mathcal{V}_{P}$ and $\mathcal{V}_{P}\left(\mathbb{R}_{>0}\right)$ is the set of totally nonnegative elements in the main open component of $\mathcal{V}_{P}$.

Note that if $P$ is a maximal parabolic subgroup, then

$$
\mathcal{V}_{P}=\left(\left(U^{\vee}\right)^{+}\right)^{e^{\vee}} \cap \overline{\left(B^{\vee}\right)^{-} w^{P}\left(B^{\vee}\right)^{-}}=\left[\left(\left(U^{\vee}\right)^{+}\right)^{e^{\vee}} \cap\left(B^{\vee}\right)^{-} w^{P}\left(B^{\vee}\right)^{-}\right] \cup\{i d\}
$$

and hence we have

$$
\mathcal{V}_{P}\left(\mathbb{R}_{\geq 0}\right)=\mathcal{V}_{P}\left(\mathbb{R}_{>0}\right) \cup\{i d\}
$$

When $P$ is the maximal parabolic subgroup $P_{n}$ of $S O_{2 n+1}(\mathbb{C})$ and $S p_{2 n}(\mathbb{C})$, or $P_{n+1}$ of $S O_{2 n+2}(\mathbb{C})$, to be consistent with the previous notation, we write, for example, $\mathcal{V}_{n}^{C}\left(\mathbb{R}_{\geq 0}\right)$ rather than $\mathcal{V}_{P_{n}}\left(\mathbb{R}_{\geq 0}\right)$ for the triple $\left(S O_{2 n+1}(\mathbb{C}), P_{n}, S p_{2 n}(\mathbb{C})\right)$.

8.2. Total positivity in Lie type A. By (8.35), we can detect totally nonnegative elements in the varieties $\mathcal{V}_{n}^{C}$ and $\mathcal{V}_{n}^{B}$ by embedding these varieties to 'relevant' varieties in Lie type $A$, whose total positivity is well understood. Now we define the 'relevant' variety in Lie type $A$ and describe its total positivity. Consider the set $\mathcal{V}_{n}:=\left\{u_{2 n}\left(t \zeta^{I}\right) \in S L_{2 n}(\mathbb{C}) \mid t \in \mathbb{C}, I \in \mathcal{T}_{n}\right\}$, with $u_{2 n}\left(t \zeta^{I}\right)$ defined in 5.1. Rietsch's result about total positivity in relation to quantum cohomology can be summarized as follows ([19]).

(1) The set $\mathcal{V}_{n}$ can be realized as a variety $\mathcal{V}_{P_{n}}$, whose coordinate ring is isomorphic to the quantum cohomology ring of the Grassmannian manifold $S L_{2 n}(\mathbb{C}) / P_{n}$, where $P_{n}$ is the maximal parabolic subgroup corresponding to the $n$-th fundamental weight.

(2) Let $\mathcal{V}_{n}\left(\mathbb{R}_{\geq 0}\right):=\left\{u_{2 n}\left(t \zeta^{I_{0}}\right) \mid t \geq 0\right\}$ and $\mathcal{V}_{n}\left(\mathbb{R}_{>0}\right):=\left\{u_{2 n}\left(t \zeta^{I_{0}}\right) \mid t \in \mathbb{R}_{>0}\right\}$. Then

$$
\begin{aligned}
& \mathcal{V}_{P_{n}}\left(\mathbb{R}_{\geq 0}\right)=\mathcal{V}_{n}\left(\mathbb{R}_{\geq 0}\right)=\left\{u_{2 n}\left(t \zeta^{I}\right) \in \mathcal{V}_{n} \mid S_{\lambda}\left(t \zeta^{I}\right) \geq 0 \text { for all } \lambda \in \mathcal{R}(n)\right\}, \\
& \mathcal{V}_{P_{n}}\left(\mathbb{R}_{>0}\right)=\mathcal{V}_{n}\left(\mathbb{R}_{>0}\right)=\left\{u_{2 n}\left(t \zeta^{I}\right) \in \mathcal{V}_{n} \mid S_{\lambda}\left(t \zeta^{I}\right)>0 \text { for all } \lambda \in \mathcal{R}(n)\right\}
\end{aligned}
$$

(3) Furthermore, the set $\mathcal{V}_{n}\left(\mathbb{R}_{>0}\right)$ is characterized by the positivity of 'special' Schur polynomials; more precisely,

$$
\mathcal{V}_{n}\left(\mathbb{R}_{>0}\right)=\left\{u_{2 n}\left(t \zeta^{I}\right) \in \mathcal{V}_{n} \mid S_{\left(m^{k}\right)}\left(t \zeta^{I}\right)>0 \text { for all } m, k \leq n\right\} .
$$

8.3. Total positivity in $\mathcal{V}_{n}^{C}$ and $\mathcal{V}_{n}^{B}$. From $\oint 8.1$ and by the analysis of the varieties $\mathcal{V}_{n}^{C}$ and $\mathcal{V}_{n}^{B}$ given in $\$ 5.2$, we now have 'natural' embeddings $\mathcal{V}_{n}^{C} \hookrightarrow \mathcal{V}_{n} \hookrightarrow$ $S L_{2 n}(\mathbb{C})$ and $\mathcal{V}_{n}^{B} \hookrightarrow S L_{2 n+1}(\mathbb{C})$, and so we will consider elements of $\mathcal{V}_{n}^{C}$ (resp. $\mathcal{V}_{n}^{B}$ ) as elements of $S L_{2 n}(\mathbb{C})$ (resp. $S L_{2 n+1}(\mathbb{C})$ ). Note that while $\mathcal{V}_{n}^{C}$ has an 'intermediate' variety $\mathcal{V}_{n}, \mathcal{V}_{n}^{B}$ does not. But an element $u_{2 n+1}\left(t \zeta^{I}\right)$ of $\mathcal{V}_{n}^{B} \subset S L_{2 n+1}$ can be viewed as a submatrix of $u_{2 n+2}\left(t \zeta^{I}\right) \in \mathcal{V}_{n+1} \subset S L_{2 n+2}(\mathbb{C})$, by taking the first $(2 n+1)$ rows and columns of $u_{2 n+2}\left(t \zeta^{I}\right)$. Therefore we can use the total positivity 
of $\mathcal{V}_{n+1}$ to describe that of $\mathcal{V}_{n}^{B}$.

$$
u_{2 n+2}\left(t \zeta^{I}\right):=\left(\begin{array}{ccccccc|c}
1 & a_{1} & a_{2} & \cdots & a_{n+1} & 0 & \cdots & 0 \\
& 1 & a_{1} & \ddots & & \ddots & & \vdots \\
& & 1 & \ddots & & & \ddots & 0 \\
& & & \ddots & & & & a_{n+1} \\
& & & & \ddots & \ddots & \ddots & \vdots \\
& & & & & 1 & a_{1} & a_{2} \\
& & & & & & 1 & a_{1} \\
\hline & & & & & & & 1
\end{array}\right),
$$

where $a_{i}=E_{i}\left(t \zeta^{I}\right)$.

Theorem 8.1. The totally nonnegative elements in $\mathcal{V}_{n}^{C}$ can be described as follows: (1) $\mathcal{V}_{n}^{C}\left(\mathbb{R}_{\geq 0}\right)=\left\{u_{2 n}\left(t \zeta^{I_{0}}\right) \mid t \in \mathbb{R}_{\geq 0}\right\}=\left\{u \in \mathcal{V}_{n}^{C} \mid \dot{\widetilde{P}}_{\lambda}(u) \geq 0\right.$ for all $\left.\lambda \in \mathcal{D}(n)\right\}$, (2) $\mathcal{V}_{n}^{C}\left(\mathbb{R}_{>0}\right)=\left\{u_{2 n}\left(t \zeta^{I_{0}}\right) \mid t \in \mathbb{R}_{>0}\right\}=\left\{u \in \mathcal{V}_{n}^{C} \mid \dot{\widetilde{P}}_{\lambda}(u)>0\right.$ for all $\left.\lambda \in \mathcal{D}(n)\right\}$.

Proof. Consider the embedding $\mathcal{V}_{n}^{C} \hookrightarrow \mathcal{V}_{n}$. Then the first identity of (1) follows from the result in type $A$ of 88.2 and (8.35). The second identity of (1) is a direct consequence of (1) of Theorem [6.6. We use the first identity of (1) and (8.37) to get the first identity of (2). The second identity of (2) follows from (1) of Theorem 6.6 and the fact that for any $I \in \mathcal{I}_{n}, \widetilde{P}_{\lambda}\left(t \zeta^{I}\right)=0$ for all $\lambda \in \mathcal{D}(n)$ if and only if $t=0$.

A similar analysis can be applied to $G=S O_{2 n+1}(\mathbb{C})$ and $\widetilde{G}=S L_{2 n+1}(\mathbb{C})$.

Theorem 8.2. The totally nonnegative elements in $\mathcal{V}_{n}^{B}$ can be described as follows: (1) $\mathcal{V}_{n}^{B}\left(\mathbb{R}_{\geq 0}\right)=\left\{u_{2 n+1}\left(t \zeta^{I_{0}}\right) \mid t \in \mathbb{R}_{\geq 0}\right\}=\left\{u \in \mathcal{V}_{n}^{B} \mid \dot{\widetilde{Q}}_{\lambda}(u) \geq 0\right.$ for all $\left.\lambda \in \mathcal{D}(n)\right\}$, (2) $\mathcal{V}_{n}^{B}\left(\mathbb{R}_{>0}\right)=\left\{u_{2 n+1}\left(t \zeta^{I_{0}}\right) \mid t \in \mathbb{R}_{>0}\right\}=\left\{u \in \mathcal{V}_{n}^{B} \mid \dot{\widetilde{Q}}_{\lambda}(u)>0\right.$ for all $\left.\lambda \in \mathcal{D}(n)\right\}$.

Proof. Since $\mathcal{V}_{n+1}\left(\mathbb{R}_{\geq 0}\right)=\left\{u_{2 n+2}\left(t \zeta^{I_{0}}\right) \mid t \in \mathbb{R}_{\geq 0}\right\}$, and since if $u_{2 n+2}\left(t \zeta^{I}\right)$ is totally nonnegative, so is the submatrix $u_{2 n+1}\left(t \zeta^{I}\right)$, then $u_{2 n+1}\left(t \zeta^{I_{0}}\right)$ for $t \geq 0$ are totally nonnegative elements of $\mathcal{V}_{n}^{B}$, i.e.,

$$
\left\{u_{2 n+1}\left(t \zeta^{I_{0}}\right) \mid t \in \mathbb{R}_{\geq 0}\right\} \subseteq \mathcal{V}_{n}^{B}\left(\mathbb{R}_{\geq 0}\right) .
$$

For the converse inclusion, considering (8.37), it suffices to show that

$$
\mathcal{V}_{n}^{B}\left(\mathbb{R}_{>0}\right) \subseteq\left\{u_{2 n+1}\left(t \zeta^{I_{0}}\right) \mid t \in \mathbb{R}_{>0}\right\} .
$$

So let $u \in \mathcal{V}_{n}^{B}\left(\mathbb{R}_{>0}\right)$. We can write $u=u_{2 n+1}\left(t_{0} \zeta^{I}\right)$ for some nonzero $t_{0} \in \mathbb{C}$ and $I \in \mathcal{I}_{n+1}$. Take $\bar{u}=u_{2 n+2}\left(t_{0} \zeta^{I}\right) \in \mathcal{V}_{n+1}$. We claim that $S_{\left(m^{k}\right)}\left(t_{0} \zeta^{I}\right)>0$ for all $m, k \leq n+1$. Firstly for $m, k$ with $m \neq n+1$, or $k \neq n+1$, by the definition of the Schur polynomials, $S_{\left(m^{k}\right)}\left(t_{0} \zeta^{I}\right)$ is the determinant of the $m \times m$ submatrix $v(m, k)$ of $u_{2 n+1}\left(t_{0} \zeta^{I}\right)$,

$$
v(m, k)=\left(\begin{array}{cccc}
a_{k} & a_{k+1} & \cdots & a_{k+m-1} \\
a_{k-1} & a_{k} & \cdots & a_{k+m-2} \\
\vdots & \vdots & & \vdots \\
a_{k-m+1} & a_{k-m+2} & \cdots & a_{k}
\end{array}\right),
$$


where $a_{i}=E_{i}\left(t_{0} \zeta^{I}\right)$ for $i>0, a_{i}=0$ for $i<0$ and $a_{0}=1$. Since $u_{2 n+1}\left(t_{0} \zeta^{I}\right)$ is totally nonnegative, the determinant of $v(m, k)$ is nonnegative. But this cannot be zero since the row vectors of $v(m, k)$ are linearly independent. Secondly, for $m, k=n+1$, we have $S_{\left(m^{k}\right)}\left(t_{0} \zeta^{I}\right)=\left(a_{n+1}\right)^{n+1}$, which is the determinant of $v(n+1, n+1)$, and positive since $a_{n+1}=E_{n+1}\left(t_{0} \zeta^{I}\right)$ is a nonzero entry of the totally nonnegative element $u_{2 n+1}\left(t_{0} \zeta^{I}\right)$. Therefore this satisfies the criterion (8.38) for $\bar{u} \in \mathcal{V}_{n+1}$ to be totally nonnegative elements. But the totally nonnegative elements of $\mathcal{V}_{n+1}$ are $u_{2 n+2}\left(t \zeta^{I_{0}}\right)$ with $t \in \mathbb{R}_{\geq 0}$, so we have $I=I_{0}$ and $t_{0}>0$, since $t_{0}$ was assumed to be nonzero. Therefore we have

$$
\mathcal{V}_{n}^{B}\left(\mathbb{R}_{>0}\right) \subseteq\left\{u_{2 n+1}\left(t \zeta^{I_{0}}\right) \mid t \in \mathbb{R}_{>0}\right\} .
$$

This proves the first identity of (1). For the second identity of (1), as in Theorem 8.1. we use Theorem 6.6 to get

$$
\left\{u_{2 n+1}\left(t \zeta^{I_{0}}\right) \mid t \in \mathbb{R}_{\geq 0}\right\}=\left\{u \in \mathcal{V}_{n}^{B} \mid \dot{\widetilde{Q}}_{\lambda}(u) \geq 0 \text { for all } \lambda \in \mathcal{D}(n+1)\right\},
$$

which is a subset of

$$
\left\{u \in \mathcal{V}_{n}^{B} \mid \dot{\widetilde{Q}}_{\lambda}(u) \geq 0 \text { for all } \lambda \in \mathcal{D}(n)\right\}
$$

So it is enough to show that

$$
\begin{aligned}
\left\{u \in \mathcal{V}_{n}^{B} \mid\right. & \left.\dot{\widetilde{Q}}_{\lambda}(u) \geq 0 \text { for all } \lambda \in \mathcal{D}(n)\right\} \\
& \subseteq\left\{u \in \mathcal{V}_{n}^{B} \mid \dot{\widetilde{Q}}_{\lambda}(u) \geq 0 \text { for all } \lambda \in \mathcal{D}(n+1)\right\}
\end{aligned}
$$

So suppose $u$ is an element of $\mathcal{V}_{n}^{B}$ such that $\dot{\widetilde{Q}}_{\lambda}(u) \geq 0$ for all $\lambda \in \mathcal{D}(n)$. Note that the relation $X_{n}^{2}=2 X_{n-1} X_{n+1}$ implies the relation

$$
\left(\dot{\widetilde{Q}}_{n}(u)\right)^{2}=2 \dot{\widetilde{Q}}_{n-1}(u) \dot{\widetilde{Q}}_{n+1}(u) .
$$

Since $\dot{\widetilde{Q}}_{n}(u)$ and $\dot{\widetilde{Q}}_{n-1}(u)$ are nonnegative, so is $\dot{\widetilde{Q}}_{n+1}(u)$. Let $\lambda \in \mathcal{D}(n+1)$. If $\lambda \in \mathcal{D}(n)$, by the assumption, $\dot{\widetilde{Q}}_{\lambda}(u)$ is nonnegative. If $\lambda \notin \mathcal{D}(n)$, we can write $\lambda=(n+1) \cup \mu$ for some $\mu \in \mathcal{D}(n)$. If we apply the factorization of $\widetilde{Q}$-polynomials, we have

$$
\dot{\widetilde{Q}}_{\lambda}(u)=\dot{\widetilde{Q}}_{n+1}(u) \dot{\widetilde{Q}}_{\mu}(u) .
$$

Since $\dot{\widetilde{Q}}_{n+1}(u)$ and $\dot{\widetilde{Q}}_{\mu}(u)$ are nonnegative, so is $\dot{\widetilde{Q}}_{\lambda}(u)$, as desired. The identities of (2) can be obtained by doing the same argument as in the proof of (2) in Theorem 8.1 .

From Theorem 8.1 and Theorem 8.2 , we conclude that Rietsch's conjecture about the relation between the total positivity and quantum cohomology ring holds for the triples $\left(S O_{2 n+1}(\mathbb{C}), S p_{2 n}(\mathbb{C}), P_{n}\right)$ and $\left(S p_{2 n}(\mathbb{C}), S O_{2 n+1}(\mathbb{C}), P_{n}\right)$.

\section{ACKNowledgments}

The author would like to thank K. Rietsch for useful correspondence about Peterson's results, A. Zelevinsky for answering many questions about the total posivity over e-mails, S. Seo for useful discussion about combinatorial aspects, and $\mathrm{H}$. Tamvakis for valuable suggestions and discussions. 


\section{REFERENCES}

[1] A. Buch, A. Kresch and H. Tamvakis, Gromov-Witten invariants on Grassmannians, J. Amer. Math. Soc. 16(2003), 901-915. MR1992829 (2004h:14060)

[2] A. Berenstein and A. Zelevinsky, Total positivity in Schubert varieties, Comment. Math. Helv. 72(1997), 128-166. MR1456321 (99g:14064)

[3] W. Fulton, Young Tableaux, London Mathematical Society, Cambridge Univ. Press, Cambridge, 1997. MR1464693 (99f:05119)

[4] W. Fulton and J. Harris, Representation Theory, Springer-Verlag, New York 1991. MR.1153249 (93a:20069)

[5] W. Fulton and R. Pandharipande, Notes on stable maps and quantum cohomology in Algebraic Geometry (Santa Cruz, 1995), 45-96, Proc. Sympos. Pure Math. 62, Part 2, Amer. Math. Soc., Providence, 1997. MR.1492534 (98m:14025)

[6] H. Hiller and B. Boe, Pieri formula for $S O_{2 n+1} / U_{n}$ and $S p_{n} / U_{n}$, Adv. in Math. 62 (1986), 49-67. MR859253 (87k:14058)

[7] B. Kostant, Flag manifold quantum cohomology, the Toda lattice, and the representation with highest weight $\rho$, Selecta Math. (N.S.) 2 (1996), 43-91. MR1403352 (97e:17029)

[8] D. Knutson, $\lambda$-rings and the representation theory of the symmetric group, Springer Lecture Notes 308, 1973. MR0364425 (51:679)

[9] A. Kresch and H. Tamvakis, Quantum cohomology of the Lagrangian Grassmannian, J. Algebraic Geometry 12 (2003), 777-810. MR.1993764 (2004h:14063)

[10] A. Kresch and H. Tamvakis, Quantum cohomology of orthogonal Grassmannians, Compositio Math. 140 (2004), 482-500. MR2027200(2005d:14080)

[11] A. Lascoux and P. Pragacz, Operator calculus for Q-Polynomials and Schubert polynomials, Adv. in Math 140 (1998), 1-43. MR1656481 (2000e:05164)

[12] G. Lusztig, Total positivity in reductive groups, Lie theory and geometry : in honor of Bertram Kostant (G. I. Lehrer, ed.), Progress in Mathematics, vol. 123, Birkhäuser, Boston, 1994, pp. 531-568. MR:1327548 (96m:20071)

[13] G. Lusztig, Total positivity and canonical bases, Algebraic groups and Lie groups, Cambridge Univ. Press, Cambridge, 1997, pp.281-295. MR.1635687 (2000j:20089)

[14] I.G. Macdonald, Symmetric Functions and Hall Polynomials, Second edition, Oxford Univ. Press, 1995. MR.1354144 (96h:05207)

[15] H. Minc, Nonnegative Matrices, John Wiley \& Sons, 1988. MR932967 (89i:15001)

[16] D. Peterson, Quantum cohomology of $G / P$, Lecture Course, Spring term, M.I.T., 1997.

[17] D. Peterson, Quantum cohomology of $G / P$, Séminaire de Mathématiques Supérieures: Representation Theories and Algebraic Geometry, Université de Montreal, Canada, July 28-Aug. 8, 1997 (unpublished lecture notes).

[18] P. Pragacz and J. Ratajski, Formulas for Lagrangian and orthogonal degeneracy loci; $\tilde{Q}$ polynomial approach, Compositio Mathematica 107; 11-87, 1997. MR1457343 (98g:14063)

[19] K. Rietsch, Quantum cohomology rings of Grassmannians and total positivity, Duke Mathematical Journal, Vol. 110, no. 3 (2001), 523-553. MR.1869115 (2003c:14063)

[20] K. Rietsch, Totally positive Toeplitz matrices and quantum cohomology of partial flag varieties, J. Amer. Math. Soc, Vol. 16(2003), 363-392 MR.1949164 (2004d:14081)

[21] K. Rietsch, A mirror symmetric construction of $H_{T}^{*}(G / P)_{(q)}$, arXiv: math.AG/0511124.

Department of Mathematics, Korea Institute for Advanced Study, 207-43 CheonGRYANGRI 2-DONG, SEOUl, 130-722, KoreA

E-mail address: daewoongc@kias.re.kr 